\title{
Revisiting Metal Fluorides as Lithium-ion Battery Cathodes
}

\author{
Xiao Hua ${ }^{1,2,3^{*}}$, Alexander S. Eggeman ${ }^{4,5}$, Elizabeth Castillo-Martínez ${ }^{1,6}$, Rosa Robert ${ }^{1}$, Harry \\ S. Geddes ${ }^{3}$, Ziheng Lü ${ }^{4}$, Chris J. Pickard ${ }^{4,7}$, Wei Meng ${ }^{1}$, Kamila M. Wiaderek ${ }^{8}$, Nathalie \\ Pereira ${ }^{9}$, Glenn G. Amatucci ${ }^{9}$, Paul A. Midgley ${ }^{4}$, Karena W. Chapman ${ }^{8,10}$, Ullrich Steiner ${ }^{2}$, \\ Andrew L. Goodwin ${ }^{3}$, and Clare P. Grey ${ }^{1 *}$
}

1: Department of Chemistry, University of Cambridge, Lensfield Road, Cambridge, CB2 1EW, United Kingdom

2: Adolphe Merkle Institute, Chemin des Verdiers 4, 1700 Fribourg, Switzerland

3: Inorganic Chemistry Laboratory, University of Oxford, South Parks Road, Oxford OX1 3QR, United Kingdom

4: Department of Materials Science and Metallurgy, University of Cambridge, Pembroke Street, Cambridge CB2 3QZ, United Kingdom

5: Department of Materials, University of Manchester, Manchester M13 9PL, United Kingdom

6. Departamento Química Inorgánica, Universidad Complutense de Madrid, 28040 Madrid, Spain

7: Advanced Institute for Materials Research, Tohoku University, Sendai, Japan

8: X-ray Science Division, Advanced Photon Source, Argonne National Laboratory, Argonne, Illinois 60439, United States

9: Energy Storage Research Group, Department of Materials Science and Engineering, Rutgers University, North Brunswick, New Jersey 08902, United States

10: Department of Chemistry, SUNY Stony Brook, Stony Brook, NY 11790-3400, United States

\begin{abstract}
Metal fluorides, promising lithium-ion battery cathode materials, have been classified as conversion materials, due to the reconstructive phase transitions widely presumed to occur upon lithiation. We challenge this view by studying $\mathrm{FeF}_{3}$ using $\mathrm{X}$-ray total scattering and electron diffraction techniques that measure structure over multiple length-scales coupled with DFT calculations, and by revisiting prior experimental studies of $\mathrm{FeF}_{2}$ and $\mathrm{CuF}_{2}$. Metal fluoride lithiation is instead dominated by diffusioncontrolled displacement mechanisms, a clear topological relationship between the metal fluoride $\mathrm{F}^{-}$ sublattices and that of $\mathrm{LiF}$ being established. Initial lithiation of $\mathrm{FeF}_{3}$ forms $\mathrm{FeF}_{2}$ on the particle's surface, along with a cation- and stacking-disordered phase, $A-\mathrm{Li}_{x} \mathrm{Fe}_{y} \mathrm{~F}_{3}$ - structurally related to $\alpha$ - $/ \beta$ $\mathrm{LiMn}^{2+} \mathrm{Fe}^{3+} \mathrm{F}_{6}$, which topotactically transforms to $B$ - and then $C-\mathrm{Li}_{x} \mathrm{Fe}_{y} \mathrm{~F}_{3}$, before forming $\mathrm{LiF}$ and $\mathrm{Fe}$. Lithiation of $\mathrm{FeF}_{2}$ and $\mathrm{CuF}_{2}$ results in a buffer phase between $\mathrm{FeF}_{2} / \mathrm{CuF}_{2}$ and $\mathrm{LiF}$. The resulting principles will aid future developments of a wider range of isomorphic metal fluorides.
\end{abstract}


In the search for lithium-ion battery (LIB) electrode materials with much higher energy densities, materials that operate via conversion (rather than topotactic (or insertion)) reactions have received considerable attention. These conversion materials can accommodate multiple electron transfers per redox centre via the equation $\mathrm{M}_{a} \mathrm{X}_{b}+(b \cdot n) \mathrm{Li}^{+}+(b \cdot n) e^{-}=a \mathrm{M}+b \mathrm{Li}_{n} \mathrm{X}(\mathrm{X}=\mathrm{F}, \mathrm{O}, \mathrm{S} \text {, etc })^{1}$, leading to large specific capacities. Because fluorine's high-electronegativity imparts a higher average voltage for reaction involving transition metal $(\mathrm{TM})$ fluorides $\left(\mathrm{MF}_{x}\right)$ than those of their oxide or sulfide counterparts ${ }^{1}$, they can be used as LIBs cathode materials ${ }^{2}$. Recent developments of fluoride-ion-based solid-state batteries ${ }^{3-7}$ and liquid fluoride ${ }^{8}$ ion-conducting electrolytes has prompted further renewed interest.

Among all the $\mathrm{MF}_{x}$ phases, $\mathrm{FeF}_{3}$ has received the most attention ${ }^{1}$ due to its low cost and toxicity ${ }^{9}$ and high energy density (1951 Wh/kg, cf. 1519 and $1874 \mathrm{Wh} / \mathrm{kg}$ for $\mathrm{FeF}_{2}$ and $\mathrm{CuF}_{2}$, respectively) ${ }^{2}$. Unlike $\mathrm{FeF}_{2} / \mathrm{CuF}_{2}, \mathrm{FeF}_{3}$ exhibits an additional high voltage plateau generally assigned to an insertion (or intercalation) process ${ }^{10,11}$ (Fig. 1a-b). This process alone shows a (practical) capacity of $\approx 200 \mathrm{mAh} / \mathrm{g}$ (cf. $\approx 170 \mathrm{mAh} / \mathrm{g}$ for both $\mathrm{LiCoO}_{2}$ and $\mathrm{LiFePO}_{4}$ ) and is largely reversible ${ }^{12}$ with a smaller overpotential than its subsequent (nominal) conversion process ${ }^{13}$ making $\mathrm{FeF}_{3}$ a promising insertion cathode. Because of its intrinsically poor electronic conductivity ${ }^{2} \mathrm{FeF}_{3}$ must be nanostructured (and embedded in a conductive matrix $)^{10,14}$ to enable good electrochemical performance. This nanosizing and the lack of long-range order on lithiation renders structure solution via X-ray diffraction (XRD) challenging ${ }^{13}$. While local probes, including solid-state nuclear magnetic resonance (NMR $)^{10}$ spectroscopy, X-ray absorption spectroscopy (XAS) $)^{11,15}$ and pair distribution function (PDF) ${ }^{10}$ analyses, have been used, no clear consensus has emerged ${ }^{11,15}$, indicating structural complexity. Numerous studies ${ }^{10,11,13,14,16-19}$ have correlated $\mathrm{FeF}_{3}$ 's phase behaviour in the insertion regime with reaction kinetics, but different mechanisms have been proposed, largely due to conflicting reports of the structure of the lithiated $\mathrm{Li}_{x} \mathrm{Fe}_{y} \mathrm{~F}_{3}$ phases. The majority of studies agree that the rhombohedrally-distorted $\mathrm{ReO}_{3}$-structure $(R \overline{3} c)$ comprising corner sharing $\mathrm{FeF}_{6}$ groups (Fig. 1c) transforms into an edge-sharing trirutile $\mathrm{Li}_{x} \mathrm{FeF}_{3}$ phase $\left(\mathrm{P}_{2} / \mathrm{mnm}\right)$, albeit with different $x$ values ${ }^{10,15,17}$, a transformation that involves considerable change in anion-packing and Fe-ordering. Less radical structural changes from $R \overline{3} c$ to $R 3 c^{11}$ or to a slightlydistorted rhombohedral phase ${ }^{13}$ have also been proposed.

To circumvent the analytical challenges, we compare the results obtained from both micro- (ca $0.2 \mathrm{um}$ ) and nano-meter (ca $7 \mathrm{~nm}$ ) $\mathrm{FeF}_{3}$ (denoted as $\mathrm{m}$ - and $\mathrm{n}-\mathrm{FeF}_{3}$, respectively), which contain similar features in their electrochemistry (Fig. 1a and S1). A charge-flipping method was applied against the electron diffraction (ED) data to solve the structures of the intermediate phases that forms on lithiating $\mathrm{m}-\mathrm{FeF}_{3}$. With this new structural insight we revisited $\mathrm{FeF}_{2}$ and $\mathrm{CuF}_{2}$ and solved the structure of the nanosized intermediate by applying our newly developed approach based on non-negative matrix factorisation (NMF) algorithm ${ }^{20}$ to PDF data, recently developed to study solid mixtures ${ }^{20}$, and which does not require any prior knowledge of the number and nature of the phases. Our results show that the general lithiation pathways in $\mathrm{FeF}_{3}$ (hexagonal close packed/hcp) and $\mathrm{FeF}_{2}$ (tetragonal close packed ${ }^{21} / t c p$ ) are dominated by topotactic diffusion-controlled displacement mechanisms. This new mechanistic insight provides a rationale for the path hysteresis ${ }^{22}$ seen in these materials.

\section{Lithiated $\mathbf{m}-\mathrm{FeF}_{3}$ structural determination}

Given the role of the initial lithiation processes in $\mathrm{FeF}_{3}$ in steering the subsequent reactions, we start by identifying the phases generated on lithiation of $\mathrm{m}-\mathrm{FeF}_{3}$. The ex situ XRD patterns of all cycled samples (Fig. $2 \mathrm{a}$ and S2) contain a new broad feature comprising two distinct components at $26.6^{\circ}$ and $28.0^{\circ}$, $2 \theta$, whose relative intensities vary with state-of-charge. Other noticeable changes include i) a large intensity reduction for the (012) reflection ascribed to Fe migration to the neighbouring sites ${ }^{19}$ and ii) the emergence of low-angle (003) and (01) reflections (indexed based on the same hexagonal lattice) indicating the loss of $c$-glide planes. Additional new reflections are seen at the states corresponding to nominal formulae $\mathrm{Li}_{0.15} \mathrm{FeF}_{3}$ and $\mathrm{Li}_{0.5} \mathrm{FeF}_{3}$, denoted as " $\mathrm{Li} 0.15$ " and "Li 0.5 ", respectively (Fig. 2b), suggesting a two-step insertion process involving the sequential formation of new phases (denoted as $A$ - and $B-\mathrm{Li}_{x} \mathrm{Fe}_{y} \mathrm{~F}_{3}$ ). These reflections are close to those of pristine $\mathrm{m}-\mathrm{FeF}_{3}$, underlying the structural correlations between $\mathrm{FeF}_{3}$ and $\mathrm{Li}_{x} \mathrm{Fe}_{y} \mathrm{~F}_{3}$. 
An assessment of the hetto- and aristo-types of (i.e., the low and high symmetry structures arising from) the $\mathrm{ABX}_{3}(R \overline{3} c)$ structure ${ }^{23}$ was made (Fig. S3) and a rhombohedral $R 3$ cell was chosen as the starting model for refinement. The refined structures for both $A$ - and $B-\mathrm{Li}_{x} \mathrm{Fe}_{y} \mathrm{~F}_{3}$ contain a near-perfect $h c p \mathrm{~F}^{-}$ lattice, with the six different crystallographic Fe sites $a_{1}-a_{6}$ (Fig. 1c) generated from symmetry reduction showing distinct $\mathrm{Fe}$ occupancies consistent with $R 3$ and $R 3 \mathrm{c}$ symmetry for the $A$ - and $B$ phases, respectively (see Sec. S1 for details); note the higher symmetry for the $B$-phase indicates an increased ordering of the Fe atoms. Based on the new symmetry, iterative refinements (Fig. S4-5) were performed and the derived structures were verified via PDF analysis (Fig. 2c-d and S6). Surprisingly, the inclusion of a considerable amount of non-crystalline rutile $\mathrm{FeF}_{2}(\sim 1.5 \mathrm{~nm})$ was required to achieve a good fit to the short-range PDF pattern of all cycled samples. The inclusion of $\mathrm{FeF}_{2}$ also accounts for the broad XRD feature at $26.6^{\circ} 2 \theta$ (Fig. S2), however, the $28.0^{\circ} 2 \theta$ scattering feature remains unmodelled, indicating that some structure/phase information is still absent in our model.

ED measurements were then performed on samples "Li 0.25 " and "Li 0.6", whose Li compositions are associated with the highest concentrations of the $A$ - and $B$-phases, respectively, allowing each phase to be studied individually. The ED data of $A$ - $\mathrm{Li}_{x} \mathrm{Fe}_{y} \mathrm{~F}_{3}$ show different systematic absences than those generated in simulations using pristine $\mathrm{FeF}_{3}(R-3 \mathrm{c})$ (Fig. $3 \mathrm{~b}$ and e) and the XRD-derived $A$-phase structure (R3) (Fig. 3c and f), with i) the emergence of $\{100\}$-type reflections as the shortest $g$-vectors (Fig. 3a) and ii) the observation of all (00l) reflections (Fig. 3d). These observations indicate that a further symmetry lowering all the way from $\mathrm{FeF}_{3}$ 's symmetry $(R-3 \mathrm{c})$ to a primitive lattice (e.g., $\left.P 3\right)$ is required to describe this phase. Furthermore, the positions with the highest intensities in the first and second row of the ED reflections parallel to the $c^{*}$ (Fig. 3d) correspond to the (013) and (023) reflections rather than (012) and (024) in $\mathrm{FeF}_{3}$, providing compelling evidence for a significant change in $\mathrm{Fe}$ positions. The charge-flipping algorithm ${ }^{24}$ (Sec. S2) was then applied using the intensities extracted from the ED pattern of an $A-\mathrm{Li}_{x} \mathrm{Fe}_{y} \mathrm{~F}_{3}$ crystal to determine the projected structure of the $A$-phase. The resulting model features "zig-zag"-arranged Fe sites (Fig. 3g). Scanning electron diffraction (SED) ${ }^{25}$, performed to evaluate the spatial distribution of the $A$-phase (Sec. S3), revealed a two-phase coexistence in one particle (Fig. $3 \mathrm{i}$ ) with the $A$-phase (Fig. $3 \mathrm{j}$ ) surrounding the pristine $\mathrm{FeF}_{3}$ phase (Fig. $3 \mathrm{k}$ ), suggesting $\mathrm{Li}_{x} \mathrm{Fe}_{y} \mathrm{~F}_{3}$ grows coherently from the parent phase. In addition, a less crystalline phase was also detected (Fig. 31) which can be indexed to rutile $\mathrm{FeF}_{2}$ (Fig. 3d). The ED data of $B-\mathrm{Li}_{x} \mathrm{Fe}_{y} \mathrm{~F}_{3}$ (Fig. $\mathrm{S} 7)$ matches the $R 3 \mathrm{c}$ model obtained from XRD with some weak additional reflections also present suggesting minor distortions, i.e., local symmetry reduction (Sec. S4).

\section{Structure and energetics of $A-\mathrm{Li}_{x} \mathrm{Fe}_{y} \mathrm{~F}_{3}$}

Density functional theory (DFT) calculations (Sec. S5) performed to evaluate any possible charge ordering in the zig-zag $A-\mathrm{Li}_{x} \mathrm{Fe}_{y} \mathrm{~F}_{3}$ structure described above, and to rationalise the weak ferromagnetism observed for this phase (Sec. S6 and Fig. S8), returned a P312 model (Table S1) with alternating $\mathrm{Fe}^{3+}$ and $\mathrm{Fe}^{2+}$ ions separated by $c / 3$ along the $c$-axis. This structure has $\mathrm{Li}$ in the $\mathrm{Fe}^{3+}$ layers (Fig. 3h) with a Fe ordering that gives rise to the strong (013) reflections seen experimentally (Fig. S9). Importantly, the overall energy of this structure is only $0.03 \mathrm{eV}$ per formula unit less stable than the thermodynamic minimum, trirutile- $\mathrm{Li}_{0.5} \mathrm{FeF}_{3}$ (Table S2). However, despite the consistency in TM ordering between the ED- and DFT-derived models, there are a few reflections/peaks (e.g., the (013)) that are either notably broadened in the experimental XRD pattern or not seen in the simulated $\mathrm{XRD/PDF}$ data (Fig. S10-11). This is ascribed to the different sample illumination sizes of the electron and X-ray scattering experiments, the larger area probed by the latter rendering the XRD/PDF-derived model an average representation of the $A$-phase. In contrast, the smaller illuminated area in ED probes the local-medium range structure, which is not necessarily affected by additional (macroscopic) structure defects.

To explain the origin of the broadening of the (013) reflection in the experimental XRD pattern, a structure incorporating stacking disorder of the $P 312$ cell (referred to as the faulted-P312 model) was implemented (Fig. 4a). In addition to the improved agreement in the XRD pattern (Fig. 4d), this faulted model can also rationalise the emergence of the $(01 l)$ and $(02 l)$ reflections in the ED patterns (Fig. S12). However, the fit to the PDF data (which represents the average local atomic structure) is still poor (see 
arrows in Fig. 4e and S11). A re-evaluation of cation ordering in the faulted-P312 model surprisingly showed that it could be constructed by using a simpler and smaller building block (Fig. 4a), with charge ordering between multivalent cations equivalent to that found in $\alpha$ - $\mathrm{LiMn}^{2+} \mathrm{Fe}^{3+} \mathrm{F}_{6}$ (Fig. 4b), differing only in (some of the) $\mathrm{Li}$ positions (i.e., $1 / 3$ of $\mathrm{Li}$ is located in the $\mathrm{TM}^{2+}$ layer in $\alpha$ - $\mathrm{LiMn}^{2+} \mathrm{Fe}^{3+} \mathrm{F}_{6}$ ). $\alpha$ $\mathrm{LiMn}^{2+} \mathrm{Fe}^{3+} \mathrm{F}_{6}$ reversibly transforms to the high-temperature $\beta$ - $\mathrm{LiMn}^{2+} \mathrm{Fe}^{3+} \mathrm{F}_{6}$ phase (both $P 321$ ) above approximately $500{ }^{\circ} \mathrm{C}$, indicating their similar energies ${ }^{26}$. The $\beta$-structure is related to the $\alpha$-form by an inversion of the $\mathrm{Li}^{+}$and $\mathrm{Fe}^{3+}$ positions. Inspired by the $\mathrm{LiMnFeF}_{6}$ dimorphism ${ }^{26}$, we built a new faulted $A$ - $\mathrm{Li}_{x} \mathrm{Fe}_{y} \mathrm{~F}_{3}$ model (Fig. 4c) incorporating disordered stacking of $\alpha$ - and $\beta$ - $\mathrm{LiFe}^{2+} \mathrm{Fe}^{3+} \mathrm{F}_{6}$ building blocks (in a ratio of 50:50) with cation orderings (including Li) mirroring those of $\alpha / \beta$ - $\mathrm{LiMn}^{2+} \mathrm{Fe}^{3+} \mathrm{F}_{6}$. The displacement vectors for the $\alpha$ - and $\beta$-segments were defined so as to minimise face-shared Fe. Given that the $\alpha / \beta$ ratio and fault probabilities are both variables, the agreement between the experiments and derived XRD and PDF simulations (Fig. 4d-e) is good. The majority of $\mathrm{Li}(5 / 6)$ resides in the $\mathrm{Fe}^{3+}$ layer in the new faulted model, still in good agreement with the DFT prediction.

\section{Insertion- and full-cycle mechanisms of $\mathbf{n}-\mathrm{FeF}_{3}$}

A careful comparison of the XRD and PDF data of $n-\mathrm{FeF}_{3}$ and $\mathrm{m}-\mathrm{FeF}_{3}$ obtained during cycling shows considerable structural similarity between the phases generated in the two systems on lithiation (Fig. 5a-c and S13). PDF analysis confirmed the presence of $A-/ B-\mathrm{Li}_{x} \mathrm{Fe}_{y} \mathrm{~F}_{3}$ and nano- $-\mathrm{FeF}_{2}$ upon lithiation of $\mathrm{n}-\mathrm{FeF}_{3}$ (Fig. 5b-c). However, unlike the two-phase pathway identified in $\mathrm{m}-\mathrm{FeF}_{3}$ for the $A-B$ phase transition, a solid-solution process from A-B was seen for $n-F_{3} F_{3}$.

We now introduce the newly identified lithiated phases into the Li-Fe-F phase diagram ${ }^{27}$, using the diagram to navigate through the intricate lithiation processes (Fig. 5d). During the initial lithiation, $\mathrm{FeF}_{3}$ undergoes a three-phase reaction (I) giving rise to $A$ - $\mathrm{Li}_{x} \mathrm{Fe}_{1-\delta} \mathrm{F}_{3}$ and $\mathrm{FeF}_{2}$ :

$$
x \mathrm{Li}+(1+2 \delta) \mathrm{FeF}_{3} \rightarrow A-\mathrm{Li}_{x} \mathrm{Fe}_{1-\delta} \mathrm{F}_{3}+3 \delta \mathrm{FeF}_{2} \quad \mathrm{I}: \text { insertion + displacement }
$$

The preservation of the $h c p$ anion framework between $\mathrm{FeF}_{3}$ and $A-\mathrm{Li}_{x} \mathrm{Fe}_{1-\delta} \mathrm{F}_{3}$ complies with a displacement-like mechanism ${ }^{28}$ with the extruded $\mathrm{FeF}_{2}$ with its $t c p$ lattice being the displaced species. One potential endpoint of this reaction is the charge-ordered phase $A-\mathrm{LiFe}^{2+} \mathrm{Fe}^{3+} \mathrm{F}_{6}(x=0.5$ and $\delta=0)$, a phase that is formed if the reaction involves insertion only. However, the $A$-phase seen electrochemically must contain Fe-deficiency $(\delta>0)$, since $\mathrm{FeF}_{2}$ formation is also observed. Note that the idealised $A$ - $\mathrm{LiFe}^{2+} \mathrm{Fe}^{3+} \mathrm{F}_{6}$ has the same composition as trirutile - the structure proposed previously ${ }^{10,15,17}$, but the $A$-phase has an $h c p$ rather than a tcp lattice.

$\mathrm{Li}$ insertion into and migration in $\mathrm{FeF}_{3}$, and the concurrent reduction of $\mathrm{Fe}^{3+}$ to $\mathrm{Fe}^{2+}$, will be relatively rapid; this, in combination with charge ordering between $\mathrm{Fe}^{2+}$ and $\mathrm{Fe}^{3+}$, will trigger the $R-3 \mathrm{c}-R 3$ rearrangement of the $\mathrm{F}$ sublattice and the nucleation and growth of the new phase $(A)$. Since the rearrangement of the $\mathrm{F}$ sublattice is only minor it will likely be associated with only a small activation barrier. In contrast, migration of the highly charged $\mathrm{Fe}^{2+/ 3+}$ ions and the significant rearrangement of the $\mathrm{F}$ sublattice both required to form $\mathrm{FeF}_{2}$ will be associated with a much higher activation barrier, and this competing reaction will be sluggish. The driving force for this reaction reflects the thermodynamic stability of the rutile sublattice ${ }^{21}$ with respect to the $h c p$ sublattice as Fe is reduced. (N.b., DFT indicates that $A$ - $\mathrm{LiFe}^{2+} \mathrm{Fe}^{3+} \mathrm{F}_{6}$ is metastable with respect to trirutile $\mathrm{LiFe}^{2+} \mathrm{Fe}^{3+} \mathrm{F}_{6}$ ). We tentatively suggest that more $\mathrm{FeF}_{2}$ was formed in the $\mathrm{m}-\mathrm{FeF}_{3}$ sample, in part because this reaction was performed at a higher temperature, but the effect of particle size on the $A-\mathrm{LiFe}^{2+} \mathrm{Fe}^{3+} \mathrm{F}_{6} / \mathrm{FeF}_{2}$ interfacial energy may also play a role. The formation of the electronically insulating layer of nano- $\mathrm{FeF}_{2}$ on the surface of $A-\mathrm{Li}_{x} \mathrm{Fe}_{1-\delta} \mathrm{F}_{3}$ results in a passivation layer, reducing contact with carbon matrix and increasing interfacial ionic resistance. This, combined with the albeit small (relative to that of $\mathrm{FeF}_{2}$ formation) activation barrier to form $A-\mathrm{Li}_{x} \mathrm{Fe}_{1-\delta} \mathrm{F}_{3}$ helps rationalise the temperature-dependent cycling behaviour where elevated temperatures $\left(\geq 60^{\circ} \mathrm{C}\right)$ were found critical - particularly for the micron phase - to reduce the overpotential in the insertion regime, ${ }^{19,29}$ these observation helps to reconcile an inconsistency in the literature - namely the observation of large overpotential for a process that is often described as a simple intercalation reaction. ${ }^{19,29}$ 
Upon further discharge, process II follows the tie line from $A-\mathrm{Li}_{x} \mathrm{Fe}_{1-\delta} \mathrm{F}_{3}$ to $B-\mathrm{Li}_{1+2 \delta} \mathrm{Fe}_{1-\delta} \mathrm{F}_{3}$ via an intercalation-type reaction with $\mathrm{Fe}$ being completely reduced to $\mathrm{Fe}^{2+}$ in $B$, a process that also involves Fe cation migration to form a Fe sublattice that complies with the higher symmetry $(R 3 c)$ :

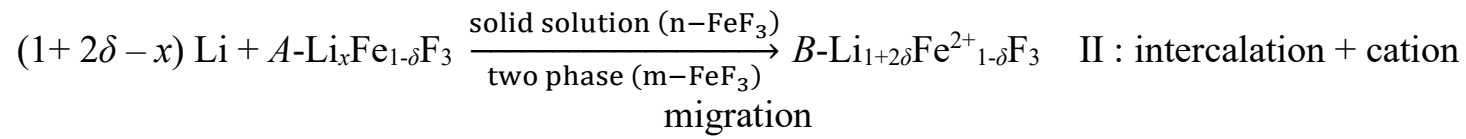

Whilst the lithiation in $\mathrm{m}-\mathrm{FeF}_{3}$ is two phase, $\mathrm{n}-\mathrm{FeF}_{3}$ undergoes a single-phase mechanism, consistent with the gradual shift in the ${ }^{6} \mathrm{Li}$ NMR resonance of this phase towards lower ppm observed in our previous ${ }^{6} \mathrm{Li} \mathrm{NMR}$ study of $\mathrm{n}-\mathrm{FeF}_{3}$ (Extended Data Fig. 1). ${ }^{10}$ This size effect has analogies with the mechanisms seen for $\mathrm{LiFePO}_{4}$ : a two phase reaction involves the formation and movement of a (high energy) interface between the two phases, which will have a higher surface area relative to the volume of the particle as particle size decreases, and thus a higher energy. ${ }^{30}$ This phenomenon can help favour a solid solution mechanism in smaller particles. The smaller polarisation in $\mathrm{n}-\mathrm{FeF}_{3}$ enables a higher completion rate of this phase transition at higher voltages (Fig. 1a), however, full capacity can be achieved at $60{ }^{\circ} \mathrm{C}$ on opening up the voltage window to $\approx 1.5 \mathrm{~V}$ (Fig. S1).

Upon completion of reaction II, a sudden voltage drop is observed signifying the end of the "insertion" discharge reaction. Subsequent charging at this point results in reactions II' and I' (the reverse of processes II and I) to reform the $A$-phase and $\mathrm{FeF}_{3}$, respectively. However, there is only a partial reformation of $\mathrm{FeF}_{3}$ (Fig. 5e) - more pronounced in micron size particles - presumably due to poor contact between $\mathrm{FeF}_{2}$ and the $A / B$-phases; this can be effectively mitigated by employing nanostructured materials and/or elevated operating temperatures, decreasing the distances over which Fe atoms have to migrate, and enhancing transport, respectively ${ }^{29}$.

In the "conversion" regime, further discharge of the $B$-phase and $\mathrm{FeF}_{2}$ proceed via separate routes. The lithiation of $B-\mathrm{Li}_{1+2 \delta} \mathrm{Fe}_{1-\delta} \mathrm{F}_{3}$ first (step III) involves the formation of another intermediate $C$ - $\mathrm{Li}_{1+2 \eta} \mathrm{Fe}^{2+}{ }_{1-}$ ${ }_{n} \mathrm{~F}_{3}$, whose structure (Sec. S7) is closely related to the $B$-phase, involving another topotactic displacement process (III). Further lithiation of the $C$-phase finally triggers the reconstruction of the $\mathrm{F}^{-}$ sublattice from $h c p$ to $f c c$ in $\mathrm{LiF}$ forming a $\mathrm{Fe}(\sim 2 \mathrm{~nm})$ and $\mathrm{LiF}(>3 \mathrm{~nm})$ composite:

$$
\begin{aligned}
& 2(\eta-\delta) \mathrm{Li}+B-\mathrm{Li}_{1+2 \delta} \mathrm{Fe}^{2+}{ }_{1-\delta} \mathrm{F}_{3} \rightarrow C-\mathrm{Li}_{1+2 \eta} \mathrm{Fe}^{2+}{ }_{1-\eta} \mathrm{F}_{3}+(\eta-\delta) \mathrm{Fe} \quad \text { III : displacement } \\
& 2(1-\eta) \mathrm{Li}+C-\mathrm{Li}_{1+2 \eta} \mathrm{Fe}_{1-\eta} \mathrm{F}_{3} \rightarrow 3 \mathrm{LiF}+(1-\eta) \mathrm{Fe} \quad \text { IV }: \text { conversion }
\end{aligned}
$$

Subsequent charge follows reversible pathways from process IV' all the way to process I' (Fig. 5d and S14). More information concerning the charge process (Sec. S8) and the second cycle (Fig. S15) is given in the SI.

\section{Mechanistic revisit of rutile fluorides}

Both the importance of $\mathrm{FeF}_{2}$ as a cathode material ${ }^{31-33}$ and our identification of two metastable $\mathrm{Li}_{1+2 x} \mathrm{Fe}_{1-}$ ${ }_{x} \mathrm{~F}_{3}$ phases situated on the $\mathrm{LiF}_{-} \mathrm{FeF}_{2}$ tie line (Extended Data Fig. 2), prompted a reanalysis of our original $\mathrm{FeF}_{2} \mathrm{PDF}$ data (Fig. S16) using a novel analytical method based on Metropolis NMF algorithm ${ }^{20}$. Our analysis (Video S1) of the first discharge cycle uncovered (at least) one additional phase in the data (Fig. 6a and S17) whose presence is supported by a weak Bragg feature at around $20^{\circ} 2 \theta$ (Fig. S16b). While a unique solution is difficult given the small size of this phase $(\sim 1.5 \mathrm{~nm})$, its atomic ordering could be well modelled using an orthorhombic $\mathrm{Li}_{2} \mathrm{FeF}_{4}(\mathrm{Cmmm})^{34}$ phase whose $(020)$ reflection coincides with the observed weak scattering feature. Notably, this structure exhibits an evident groupsubgroup relationship ${ }^{23}$ with the reactant $\left(P 4_{2} / m n m\right)$ and the LiF product $(F m \overline{3} m)$ (Fig. 6b). Inclusion of this $\mathrm{Li}_{2} \mathrm{FeF}_{4}$ phase in our refinements leads to a significantly improved fit (Fig. S18c) with the refined crystallite size almost constant $(\sim 1.5 \mathrm{~nm})$ during the whole cycle. In addition, $\mathrm{Li}_{2} \mathrm{FeF}_{4}$ is also identified in $\mathrm{FeF}_{2}$ 's charge process (Fig. S18b) and at the end of the discharge/early in the charge of $\mathrm{FeF}_{3}$ (Fig. $\mathrm{S} 18 \mathrm{e}-\mathrm{f}$ ) - likely via a reaction involving extruded $\mathrm{FeF}_{2}$. The results imply that the intermediate $\mathrm{Li}_{2} \mathrm{FeF}_{4}$ is present - at least in part - as an interface, whose formation is likely to mitigate the substantial 
structural difference between the reactant $\mathrm{FeF}_{2}$ and product $\mathrm{LiF}$. To help validate this hypothesis, we constructed an interface model $\left(\mathrm{LiF}_{\mid}\left|\mathrm{Li}_{2} \mathrm{FeF}_{4}\right| \mathrm{FeF}_{2}\right)$ in which $\mathrm{Li}_{2} \mathrm{FeF}_{4}$ is coherently connected to $\mathrm{LiF}$ and $\mathrm{FeF}_{2}$ units according to their underlying symmetry relationships (Fig. 6c). Based our DFT studies of this interface model, as well as an evaluation of the energies of the related phases under lattice matching conditions (Fig. S19), we conclude that the inclusion of the $\mathrm{Li}_{2} \mathrm{FeF}_{4}$ interfacial component is energetically favoured (Sec. S9). Therefore, although $\mathrm{FeF}_{2}$ has been widely perceived to function via conversion mechanism, our analyses point to a reversible two-step topotactic displacement pathway $\left(\mathrm{V}_{\mathrm{I}}\right.$ and $\left.\mathrm{V}_{\mathrm{II}}\right)$. We have further verified that a similar mechanism occurs in $\mathrm{CuF}_{2}(\mathrm{Sec} .10$ and Video $\mathrm{S} 2)$ and have identified an orthorhombic $\mathrm{Li}_{2} \mathrm{CuF}_{4}(\mathrm{Cmca})^{34}$ phase as the intermediate phase, with a different $\mathrm{Li} / \mathrm{Cu}$ ordering scheme (Fig. S20-22 for full details). The idealised and simplified reactions can, therefore, be written as:

$$
\begin{aligned}
2 x \mathrm{Li}+\mathrm{MF}_{2} \rightarrow \mathrm{Li}_{2 x} \mathrm{M}_{1-x} \mathrm{~F}_{2}+x \mathrm{M} & (\mathrm{M}=\mathrm{Fe}, \mathrm{Cu} ; 0<x \leq 0.5) \mathrm{V}_{\mathrm{I}} \text { : displacement } \\
2 \mathrm{Li}+\mathrm{Li}_{2} \mathrm{MF}_{4} \rightarrow 4 \mathrm{LiF}+\mathrm{M} & (\mathrm{M}=\mathrm{Fe}, \mathrm{Cu}) \quad \mathrm{V}_{\mathrm{II}}: \text { displacement }
\end{aligned}
$$

We note that earlier TEM studies also reported a topotactic lithiation of $\mathrm{FeF}_{2}{ }^{31}$, but despite sustained efforts $^{31-33,35,36}$, and with the exception of our earlier ${ }^{6} \mathrm{Li} \mathrm{NMR} \mathrm{study}{ }^{10}$, the presence of an intermediate was not confirmed in other studies of the bulk phases. The results presented here differ from those in a recent TEM study ${ }^{32}$ which reported the formation of an $\mathrm{Fe}^{3+}$-containing trirutile intermediate phase $\left(\mathrm{LiFe}^{2+} \mathrm{Fe}^{3+} \mathrm{F}_{6}\right)$ upon lithiation of $\mathrm{FeF}_{2}$. We note that the high sensitivity of $\mathrm{FeF}_{x}$ to electron radiation damage $^{37}$ at high dose rates ${ }^{32}$ (explored further in the Sec. S11) highlights the need to ensure that the structural models proposed with TEM data are consistent with the results acquired from multiple structural techniques (Fig. S23-24). Given the structure flexibility of these orthorhombic structures in accommodating symmetry changes and lattice-/atomic distortions during the $t c p$ (rutile)- $f c c$ (rocksalt) transition, we infer that a number of other rutile-related TM fluorides may follow a similar displacement process. Further experimental and computation studies are needed to understand the role that these buffer phases play in controlling reactivity and rates.

\section{Towards a comprehensive account of $\mathrm{FeF}_{3}-\mathrm{FeF}_{2}$ system}

Based on the improved mechanistic knowledge of rutile's phase behaviour upon cycling, we now consider the relationship between $\mathrm{FeF}_{3}$ and $\mathrm{FeF}_{2}$ given their coexistence during the whole $\mathrm{FeF}_{3}$ cycle. It is worth noting that while some studies also observed $\mathrm{FeF}_{2}{ }^{15,29}$ upon lithiation of $\mathrm{FeF}_{3}$, the formation of this rutile phase derived from a phase transition of the whole $\mathrm{FeF}_{3}$ particle. However, we stress that the original $h c p \mathrm{~F}^{-}$lattice is largely retained all the way to the last lithiation step to form $f c c \mathrm{LiF}$. The $\mathrm{F}^{-}$ lattice transition from $h c p\left(\mathrm{FeF}_{3}\right)$ to tcp (rutile) only occurs at the surface of the $\mathrm{FeF}_{3}$ particle. Furthermore, in contrast to the occurrence of an intermediate stage in $\mathrm{FeF}_{2}$ 's lithiation to form $\mathrm{LiF}$ ( + $\mathrm{Fe}$ ) via a $t c p-f c c$ lattice transition (reaction $\mathrm{V}_{\mathrm{I}}$ and $\mathrm{V}_{\mathrm{II}}$ ), the $h c p-f c c$ transformation in the lithiation of $C$ $\mathrm{Li}_{1+2 \eta} \mathrm{Fe}_{1-\eta} \mathrm{F}_{3}$ (reaction IV) proceeds via a reconstructive conversion reaction. A fcc-hcp reconversion takes place concurrently with the $f c c$-tcp transition during the charge of $\mathrm{FeF}_{3}$, while only the latter was observed in $\mathrm{FeF}_{2}$ (within the same voltage window). Significantly more $\mathrm{FeF}_{2}$ relative to $\mathrm{Li}_{x} \mathrm{Fe}_{y} \mathrm{~F}_{3}$ is formed upon charge of the $\mathrm{FeF}_{3}$ system, suggesting that the topotactic $f c c$-tcp transition is the favoured delithiation process. This work suggests that the nature of the interfaces may play a role in controlling the amount of $\mathrm{FeF}_{3}\left(\mathrm{cf} \mathrm{FeF}_{2}\right)$ upon charge, an important factor which likely needs to be considered in the preparation of $\mathrm{FeF}_{3} / \mathrm{FeF}_{2}$ electrodes using pre-lithiated $\mathrm{LiF} / \mathrm{Fe}$ mixtures ${ }^{38-40}$.

As a final point, we highlight that the lithiation and delithiation steps for both $\mathrm{FeF}_{3}$ and $\mathrm{MF}_{2}$ are primarily diffusion-controlled processes dominated by displacement mechanisms, whose kinetic behaviour mainly depends on the mobility difference between the displaced species. ${ }^{22}$ During discharge, the concurrent $\mathrm{Li}$ insertion and $\mathrm{M}$ extrusion is limited by the TM diffusion due to the generally faster $\mathrm{Li}$ transport, resulting in almost invariant overpotentials, hence flat operating voltages; while upon charge, a more rapid Li removal than $\mathrm{M}$ insertion leads to gradually increased overpotentials as $\mathrm{Li}$ is extracted, resulting in more sloping voltage profiles. This phenomenon has been described as "path hysteresis" and is characteristic for displacement reactions. ${ }^{22}$ Hence, while structure engineering 
remains important to improve the rate performance, we note that effective mitigation of intrinsic path hysteresis will require exploitation of material systems with improved metal (ion) mobilities.

To summarise, we have performed a comprehensive investigation of $\mathrm{FeF}_{3}$ 's structural behaviour as a cathode material for LIBs and revisited our previous work on $\mathrm{FeF}_{2}$ and $\mathrm{CuF}_{2}{ }^{41}$. We demonstrated the need to obtain crystallographic information over a wide range of length scales to develop a compelling atomistic model that could be tested against all the data. Our study revealed an unexpectedly complicated lithiation process during $\mathrm{FeF}_{3}$ 's initial discharge, involving displacement of nanoscale $\mathrm{FeF}_{2}$ and concurrent formation of $A-\mathrm{Li}_{x} \mathrm{Fe}_{y} \mathrm{~F}_{3}$, whose intricate structure contains faulted stacking of motifs that are isostructural to $\alpha$ - and $\beta$ - $\mathrm{LiMnFeF}_{6}$. Further lithiation of the $A$-phase and $\mathrm{FeF}_{2}$ proceed via distinct routes that are dominated by displacement reactions based on topological relationships between these fluorides' $\mathrm{F}^{-}$lattices $(h c p / t c p)$ and that in the rocksalt product $(f c c)$. This renewed mechanistic insight reveals an underlying principle that may serve as a reference model for a wider range of isomorphic binary fluorides and ternary systems ${ }^{36,42}$; it also rationalises the intrinsic path hysteresis, suggesting that it could be mitigated by employing displaced species with enhanced mobilities. From a methodological perspective, this work demonstrates the viability of our novel analytical approach combining PDF and NMF to uncover minor species within a heterogenous system, with broader implications beyond energy storage.

\section{REFERENCES}

1 Cabana, J., Monconduit, L., Larcher, D. \& Palacín, M. R. Beyond Intercalation-Based Li-Ion Batteries: The State of the Art and Challenges of Electrode Materials Reacting Through Conversion Reactions. Adv. Mater. 22, E170-E192 (2010).

2 Amatucci, G. G. \& Pereira, N. Fluoride based electrode materials for advanced energy storage devices. J. Fluorine Chem. 128, 243-262 (2007).

3 Grenier, A. et al. Solid Fluoride Electrolytes and Their Composite with Carbon: Issues and Challenges for Rechargeable Solid State Fluoride-Ion Batteries. J. Phys. Chem. C 121, 2496224970 (2017).

4 Thieu, D. T. et al. $\mathrm{CuF}_{2}$ as Reversible Cathode for Fluoride Ion Batteries. Adv. Funct. Mater. 27, 1701051 (2017).

$5 \quad$ Zhang, L., Anji Reddy, M. \& Fichtner, M. Development of tysonite-type fluoride conducting thin film electrolytes for fluoride ion batteries. Solid State Ion. 272, 39-44 (2015).

6 Anji Reddy, M. \& Fichtner, M. Batteries based on fluoride shuttle. J. Mater. Chem. 21, 1705917062 (2011).

7 Gschwind, F. et al. Fluoride ion batteries: Theoretical performance, safety, toxicity, and a combinatorial screening of new electrodes. J. Fluorine Chem. 182, 76-90 (2016).

8 Davis, V. K. et al. Room-temperature cycling of metal fluoride electrodes: Liquid electrolytes for high-energy fluoride ion cells. Science 362, 1144 (2018).

9 Reddy, M. A. \& Fichtner, M. in Advanced Fluoride-Based Materials for Energy Conversion (ed Henri Groult) 51-76 (Elsevier, 2015).

10 Yamakawa, N., Jiang, M., Key, B. \& Grey, C. P. Identifying the Local Structures Formed during Lithiation of the Conversion Material, Iron Fluoride, in a Li Ion Battery: A Solid-State NMR, X-ray Diffraction, and Pair Distribution Function Analysis Study. J. Am. Chem. Soc. 131, 10525-10536 (2009).

11 Zhang, W. et al. In Situ Electrochemical XAFS Studies on an Iron Fluoride High-Capacity Cathode Material for Rechargeable Lithium Batteries. J. Phys. Chem. C 117, 11498-11505 (2013).

12 Kim, S.-W., Seo, D.-H., Gwon, H., Kim, J. \& Kang, K. Fabrication of FeF 3 Nanoflowers on CNT Branches and Their Application to High Power Lithium Rechargeable Batteries. Adv. Mater. 22, 5260-5264 (2010).

13 Tan, H. J. et al. Electrochemical Cycling and Lithium Insertion in Nanostructured $\mathrm{FeF}_{3}$ Cathodes. J. Electrochem. Soc. 161, A445-A449 (2014). 
14 Badway, F., Cosandey, F., Pereira, N. \& Amatucci, G. G. Carbon Metal Fluoride Nanocomposites. J. Electrochem. Soc. 150, A1318 (2003).

15 Li, L. et al. Origins of Large Voltage Hysteresis in High-Energy-Density Metal Fluoride Lithium-Ion Battery Conversion Electrodes. J. Am. Chem. Soc. 138, 2838-2848 (2016).

16 Liu, P., Vajo, J. J., Wang, J. S., Li, W. \& Liu, J. Thermodynamics and Kinetics of the Li/FeF 3 Reaction by Electrochemical Analysis. J. Phys. Chem. C 116, 6467-6473 (2012).

17 Li, L. et al. Visualization of electrochemically driven solid-state phase transformations using operando hard X-ray spectro-imaging. Nat. Commun. 6, 6883 (2015).

18 Zhou, M., Zhao, L., Kitajou, A., Okada, S. \& Yamaki, J.-i. Mechanism on exothermic heat of $\mathrm{FeF}_{3}$ cathode in Li-ion batteries. J. Power Sources 203, 103-108 (2012).

19 Badway, F., Pereira, N., Cosandey, F. \& Amatucci, G. G. Carbon-Metal Fluoride Nanocomposites: Structure and Electrochemistry of $\mathrm{FeF}_{3}$ : C. J. Electrochem. Soc. 150, A1209A1218 (2003).

20 Geddes, H. S., Blade, H., McCabe, J. F., Hughes, L. P. \& Goodwin, A. L. Structural characterisation of amorphous solid dispersions via metropolis matrix factorisation of pair distribution function data. Chem. Commun. 55, 13346-13349 (2019).

21 West, A. R. \& Bruce, P. G. Tetragonal-packed crystal structures. Acta Crystallogr. B 38, 18911896 (1982).

$22 \mathrm{Yu}, \mathrm{H}$. C. et al. Designing the next generation high capacity battery electrodes. Energy Environ. Sci. 7, 1760-1768 (2014).

23 Müller, U. Symmetry relationships between crystal structures: applications of crystallographic group theory in crystal chemistry. Vol. 18 (OUP Oxford, 2013).

24 Oszlanyi, G. \& Suto, A. Ab initio structure solution by charge flipping. Acta Crystallogr. A 60, 134-141 (2004).

25 Rauch, E. et al. Automatic crystal orientation and phase mapping in TEM by precession diffraction. Microscopy and Analysis 22, S5-S8 (EU) (2008).

26 Courbion, G., Jacoboni, C. \& De Pape, R. The dimorphism of $\mathrm{LiMnFeF}_{6}$ : A new kind of cationic order in the structural type $\mathrm{Na}_{2} \mathrm{SiF}_{6}$. J. Solid State Chem. 45, 127-134 (1982).

27 Doe, R. E., Persson, K. A., Meng, Y. S. \& Ceder, G. First-Principles Investigation of the $\mathrm{Li}-\mathrm{Fe}-\mathrm{F}$ Phase Diagram and Equilibrium and Nonequilibrium Conversion Reactions of Iron Fluorides with Lithium. Chem. Mater. 20, 5274-5283 (2008).

28 Goodenough, J. B. \& Park, K.-S. The Li-Ion Rechargeable Battery: A Perspective. J. Am. Chem. Soc. 135, 1167-1176 (2013).

29 Tawa, S., Matsumoto, K. \& Hagiwara, R. Reaction Pathways of Iron Trifluoride Investigated by Operation at $363 \mathrm{~K}$ Using an Ionic Liquid Electrolyte. J. Electrochem. Soc. 166, A2105A2110 (2019).

30 Van der Ven, A., Garikipati, K., Kim, S. \& Wagemaker, M. The Role of Coherency Strains on Phase Stability in $\mathrm{Li}_{x} \mathrm{FePO}_{4}$ : Needle Crystallites Minimize Coherency Strain and Overpotential. J. Electrochem. Soc. 156, A949 (2009).

31 Karki, K. et al. Revisiting Conversion Reaction Mechanisms in Lithium Batteries: LithiationDriven Topotactic Transformation in $\mathrm{FeF}_{2}$. J. Am. Chem. Soc. 140, 17915-17922 (2018).

32 Xiao, A. W. et al. Understanding the conversion mechanism and performance of monodisperse $\mathrm{FeF}_{2}$ nanocrystal cathodes. Nat. Mater. 19, 644-654 (2020).

33 Wang, F. et al. Conversion Reaction Mechanisms in Lithium Ion Batteries: Study of the Binary Metal Fluoride Electrodes. J. Am. Chem. Soc. 133, 18828-18836 (2011).

34 Jain, A. et al. Commentary: The Materials Project: A materials genome approach to accelerating materials innovation. APL Materials 1, 011002 (2013).

35 Wang, F. et al. Tracking lithium transport and electrochemical reactions in nanoparticles. Nat. Commun. 3, 1201 (2012).

36 Wang, F. et al. Ternary metal fluorides as high-energy cathodes with low cycling hysteresis. Nat. Commun. 6, 6668 (2015).

37 Cosandey, F., Al-Sharab, J. F., Badway, F., Amatucci, G. G. \& Stadelmann, P. EELS Spectroscopy of Iron Fluorides and $\mathrm{FeF}_{x} / \mathrm{C}$ Nanocomposite Electrodes Used in Li-Ion Batteries. Microsc. Microanal. 13, 87-95 (2007). 
38 Sun, Y. et al. In Situ Chemical Synthesis of Lithium Fluoride/Metal Nanocomposite for High Capacity Prelithiation of Cathodes. Nano Lett. 16, 1497-1501 (2016).

39 Hori, H. \& Okada, S. Reconversion Reaction of LiF/Fe Composite Thin Film Cathodes for Lithium-Ion Battery. Electrochemistry 83, 909-913 (2015).

40 Li, T., Chen, Z. X., Ai, X. P., Cao, Y. L. \& Yang, H. X. LiF/Fe nanocomposite as a lithiumrich and high capacity conversion cathode material for Li-ion batteries. J. Power Sources 217, 54-58 (2012).

41 Hua, X. et al. Comprehensive Study of the $\mathrm{CuF}_{2}$ Conversion Reaction Mechanism in a Lithium Ion Battery. J. Phys. Chem. C 118, 15169-15184 (2014).

42 Huang, Q. et al. Fading Mechanisms and Voltage Hysteresis in $\mathrm{FeF}_{2}-\mathrm{NiF}_{2}$ Solid Solution Cathodes for Lithium and Lithium-Ion Batteries. Small 15, 1804670 (2019). 


\section{METHODS}

Materials preparation. $\mathrm{m}_{-} \mathrm{FeF}_{3}$ electrode mixtures were prepared by mixing $75 w t \% \mathrm{FeF}_{3}$ (sigmaaldrich) and $25 \mathrm{wt} \%$ carbon manually ground in an agate mortar. $\mathrm{n}-\mathrm{FeF}_{3}, \mathrm{FeF}_{2}$, and $\mathrm{CuF}_{2}$ nanocomposites were prepared as described elsewhere ${ }^{41,43}$. Their electrode mixtures were prepared by mixing $90 w t \%$ of the nanocomposite with an additional $10 w t \%$ of carbon that were manually ground in an Agathe mortar. The resulting loose powders were directly used as cathodes without any additional binder. Swagelok cells were assembled with 3-5 mg of cathode powders, a Whatman glass fibre separator wetted with electrolyte and a Li metal disk. The electrolyte was $1 \mathrm{M} \mathrm{LiPF}_{6}$ in $1: 1(\mathrm{v} / \mathrm{v})$ EC/DMC. Electrochemical tests were carried out using a Bio-Logic potentiostat. Samples for HRXRD, PDF, ED, and SQUID were recovered from batteries stopped at the selected states of charge, disassembled inside the glovebox and rinsed with DMC twice before drying and filling the capillaries or SQUID capsule. All the charged samples (apart from the ones at the early charge) were prepared at selected voltages because using voltage criterion to choose critical state of charge is a more reliable experimental control at late charge. A drop of the DMC suspension was used for TEM grid preparation. TEM grids were sealed under Argon until transferred into the TEM.

X-ray diffraction and total scattering. Ex situ HRXRD experiments were performed at the Advanced Photon Source (APS) beamline 11-BM $(\lambda=0.4136 \AA)$ using a 12-channel analyzer detector array. Ex situ X-ray total scattering were performed at APS beamline 11-ID-B $(\lambda=0.2127 \AA)$ and Diamond beamline I15-1 $(\lambda=0.1617 \AA)$ both using an area detector. PDF patterns $\left(Q_{\max }=24 \AA^{-1}\right)$ were generated using Fit $2 \mathrm{D}^{44} / \mathrm{DAWN} \mathrm{N}^{45}$ and PDFgetX $2^{46}$ for data reduction and normalisation, respectively. All samples were sealed in either Kapton or quartz capillaries. XRD and PDF analyses were performed using TOPAS v4 / GSAS-II ${ }^{47}$ and PDFgui ${ }^{48}$ / DISCUS ${ }^{49}$ programs respectively. Stacking faults was modelled using DIFFaX ${ }^{50}$. In situ X-ray total scattering data collection for $\mathrm{CuF}_{2}$ is described elsewhere ${ }^{41}$.

Electron diffraction. Electron diffraction experiments were performed on a Philips CM30 TEM operating at $300 \mathrm{kV}$. A Nanomegas Spinningstar apparatus was used to produce a beam precession angle of $1^{\circ}$. Images were recorded on Ditabis imaging plates and processed using software developed in the Electron Microscopy Group in Cambridge. Scanning electron diffraction experiments were performed on a Philips CM300 FEGTEM operating at 300kV equipped with a Nanomegas Digistar apparatus to generate a scan area of $275 \mathrm{~nm} \times 275 \mathrm{~nm}$ with a scan step of $2.5 \mathrm{~nm}$. Diffraction patterns were recorded on a high speed external digital camera and phase and orientation analysis was performed using the ASTAR software package.

SQUID. Magnetization was measured on a Quantum Design Magnetic Properties Measurement System (MPMS) with a superconducting quantum interference device (SQUID) magnetometer. Zero Field cooled (ZFC) Field cooled (FC) (100 Oe) magnetic susceptibility $\chi(\mathrm{T})$ was measured in the temperature range 2-300K. Magnetization vs Magnetic field $(\mathrm{H})$ measurements were recorded in the $0-5$ Tesla range at $200 \mathrm{~K}$ on pristine $\mathrm{FeF}_{3}$ and dis $\mathrm{Li} 0.4$.

Density functional theory calculation. Combined crystal and magnetic structure predictions were performed using $\mathrm{Ab}$ Initio Random Structure Searching (AIRSS) ${ }^{51,52}$. Energy optimisations were performed using CASTEP ${ }^{10}$. In order to remain consistent with the composition of the trirutile phase $\left(P 4_{2} / \mathrm{mnm}^{10}{ }^{10}\right.$, calculations were performed for a stoichiometry of $\mathrm{Li}_{0.5} \mathrm{FeF}_{3}$ and the cell parameters were adopted from the HRXRD refinement. The calculation was performed using a $700 \mathrm{eV}$ plane-wave cutoff energy and a $5 \times 5 \times 2$ Monkhorst-Pack $k$-point grid using the PBE functional. GGA+U was used with a $\mathrm{U}$ on the iron $d$-channel of $4 \mathrm{eV}$. Full computational details are available as part of the supplied computed data. The energy calculation for the interface model was performed using the same setting.

Non-negative matrix factorisation. The NMF approach followed closely the Metropolis Matrix Factorisation (MMF) method reported earlier ${ }^{20}$, which uses the MMC algorithm to carry out $\mathrm{NMF}^{53}$. The NMF analysis was performed on renormalised PDFs to satisfy the non-negative criterion of NMF. 
The renormalised $g^{\exp }(r)$ were derived from the experimental $G^{\exp }(r)$ using equation $G(r)=$ $4 \pi r \rho_{0}(\mathrm{~g}(r)-1)^{54}$, in which $\rho_{0}$ refers to the number density of the structure model. Three fundamental components $g_{i}^{*}(r)(i=4)$ were employed in the analysis. The goal of the analysis was to identify these $g_{i}^{*}(r)$ and associated weights $w_{i j}\left(j\right.$ corresponds to the number of experimental $\left.g_{j}^{\exp }(r)\right)$ to minimise $\left|g_{j}^{\text {calc }}(r)-g_{j}^{\text {exp }}(r)\right|^{2}$, where $g_{j}^{\text {calc }}(r)=\sum_{i=1}^{3} w_{i j} g_{i}^{*}(r)$. Additional constraints were applied to ensure nonnegative $g_{i}^{*}(r)$ for all $i$ and $r$, and that $\sum_{i=1}^{4} w_{i j}=1$ for all $j$. The initial $g_{l}^{*}(r)$ and $g_{2}^{*}(r)$ representing the known components that were fixed to respectively represent $\mathrm{FeF}_{2} / \mathrm{CuF}_{2}$ and $\mathrm{Fe} / \mathrm{Cu}$, whereas the two unknown components $g_{3}^{*}(r)$ and $g_{4}^{*}(r)$ and all $w_{i j}$ were assigned randomly subject to the various constraints listed above. Each iteration involved random variation of these parameters, followed by the calculation of the change in $\left|g_{j}^{\text {calc }}(r)-g_{j}^{\exp }(r)\right|^{2}$. The acceptance or rejection of the variation follows MMC algorithm. The variation was repeated under increasingly stringent acceptance criteria using simulated annealing until convergence was achieved.

\section{Data availability}

The authors declare that all data supporting the findings of this study are included within the paper and its Supplementary Information files. Source data are available from the corresponding authors upon reasonable request.

\section{Reference:}

43 Pereira, N., Badway, F., Wartelsky, M., Gunn, S. \& Amatucci, G. G. Iron Oxyfluorides as High Capacity Cathode Materials for Lithium Batteries. J. Electrochem. Soc. 156, A407 (2009).

44 Hammersley, A. FIT2D V9.129 reference manual V3.1. ESRF internal report 98HA01T. ESRF (1998).

45 Basham, M. et al. Data Analysis WorkbeNch (DAWN). J. Synchrotron Radiat. 22, 853-858 (2015).

46 Qiu, X., Thompson, J. W. \& Billinge, S. L. J. J. Appl. Crystallogr. 37, 678 (2004).

47 Toby, B. H. \& Von Dreele, R. B. GSAS-II: the genesis of a modern open-source all purpose crystallography software package. J. Appl. Crystallogr. 46, 544-549 (2013).

48 Farrow, C. L. et al. PDFfit2 and PDFgui: computer programs for studying nanostructure in crystals. J. Phys.: Condens. Matter 19, 335219 (2007).

49 Proffen, T. \& Neder, R. B. DISCUS, a program for diffuse scattering and defect structure simulations - update. J. Appl. Crystallogr. 32, 838-839 (1999).

50 Treacy, M. M. J., Newsam, J. M. \& Deem, M. W. A General Recursion Method for Calculating Diffracted Intensities from Crystals Containing Planar Faults. Proc. Math. Phys. Eng. Sci. 433, 499-520 (1991).

51 Pickard, C. J. \& Needs, R. J. High-Pressure Phases of Silane. Phys. Rev. Lett. 97, 045504 (2006).

52 Pickard, C. J. \& Needs, R. J. Ab initiorandom structure searching. J. Phys.: Condens. Matter 23, 053201 (2011).

53 Lee, D. D. \& Seung, H. S. Learning the parts of objects by non-negative matrix factorization. Nature 401, 788-791 (1999).

54 Egami, T. \& Billinge, S. J. L. Underneath the Bragg Peaks Structural Analysis of Complex Materials. (Pergamon, 2012). 


\section{Acknowledgements}

X.H. is supported by funding from EPSRC Doctoral Prize, Adolphe Merkle and the Swiss National Science Foundation (Program NRP70 No. 153990) and European Commission via MSCA (Grant 798169). A.S.E. acknowledges financial support from the Royal Society. E.C.M. acknowledges funding from European Commission via MSCA (Grant 747449) and RTI2018-094550-A-100 from MICINN. Z. L. acknowledges funding from the Faraday Institution via the FutureCat consortium. C.J.P. is supported by the Royal Society through a Royal Society Wolfson Research Merit award, and EPSRC grant EP/P022596/1. A.L.G. acknowledges funding from the ERC (Grant 788144). This research was supported as part of the North Eastern Center for Chemical Energy Storage, an Energy Frontier Research Center funded by the US Department of Energy, Office of Science, and Office of Basic Energy Sciences under Award Number DE-SC0001294. Work done at Argonne and use of the Advanced Photon Source, an Office of Science User Facility operated for the US Department of Energy (DOE) Office of Science by Argonne National Laboratory, was supported by the US DOE under Contract No. DE-AC02-06CH11357. Work done at Diamond Light Source was under Proposal EE17315-1. The authors are grateful to Prof. G. Ceder and other NECCES members for many stimulating discussions concerning fluoride-based conversion reactions and on the origins of structural hysteresis. The authors also acknowledge the help from S. Dutton, T. Dean, A. Docker, M. Leskes and D. Keeble.

\section{Author contributions}

X.H., E.C.M. and R.R. planned the project with C.P.G. E.C.M. and R.R. prepared the pristine materials with help from N.P. and G.G.A. X.H., E.C.M., R.R. and M.W. performed the electrochemistry tests and prepared samples for ex situ characterisation. E.C.M. and R.R. performed HRXRD measurements. A.S.E. acquired and analysed TEM data with support from P.A.M. X.H., R.R. and K.M.W. acquired PDF data with support from U.S. and K.W.C. X.H. performed analyses of the electrochemistry, XRD and PDF data. Z.L. and C.J.P. performed the DFT calculations. X.H., H.S.G. and A.L.G. performed the NMF analysis. X.H. and C.P.G. wrote the manuscript with the input from all the coauthors.

\section{Corresponding author}

Correspondence to Xiao Hua and Clare P. Grey.

\section{Competing interests}

The authors declare no competing interests.

\section{Supplementary information}

Sections on initial XRD refinement (S1), charge-flipping (S2), scanning electron diffraction (S3), ED analysis of $B-\mathrm{Li}_{x} \mathrm{Fe}_{y} \mathrm{~F}_{3}$ (S4), DFT calculation (S5), SQUID magnetometry data (S6), crystal structure of $\mathrm{C}-\mathrm{Li}_{1+2 \eta} \mathrm{Fe}_{1-\eta} \mathrm{F}_{3}$ and its lithiation (S7), charge process mechanism (S8), energy study of the interface model (S9), NMF study of lithiated $\mathrm{CuF}_{2}(\mathrm{~S} 10)$ and radiation sensitivity of $\mathrm{FeF}_{x}(\mathrm{~S} 11)$, Figure $\mathrm{S} 1-\mathrm{S} 24$, Table S1 - S2, Video S1 - S2 and full details on DFT computation (compressed file). 


\section{FIGURES}

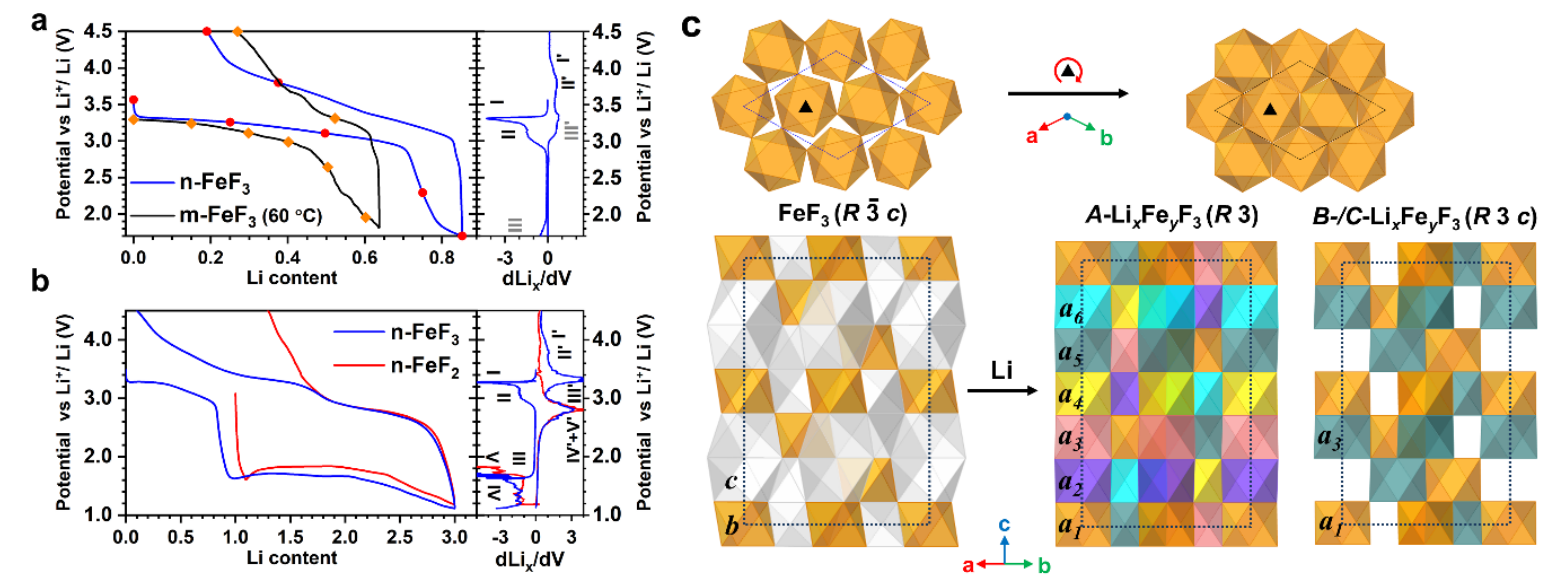

Fig. 1 | Electrochemical performance and crystal structures. Comparison of galvanostatic profiles between a) $n-\mathrm{FeF}_{3}$ (blue) and $\mathrm{m}-\mathrm{FeF}_{3}$ (black) within the insertion cycle, and between $\mathbf{b}$ ) $\mathrm{n}-\mathrm{FeF}_{3}$ (blue) and $\mathrm{n}-\mathrm{FeF}_{2}(\mathrm{red})$ in the full reaction cycle. The corresponding derivative curves $\left(d \mathrm{Li}_{x} / d \mathrm{~V}\right)$ from $\mathrm{n}-\mathrm{FeF}_{3}$ are shown with the key reactions labelled. The states of charge during the insertion cycle where X-ray scattering experiments were performed are marked. c) Top: projection of $\mathrm{FeF}_{3}$ and $\mathrm{Li}_{x} \mathrm{Fe}_{y} \mathrm{~F}_{3}$ along the $c$-axis. The black triangles denote a 3 -fold axis around which a clockwise rotation of the $\mathrm{FeF}_{6}$ octahedron leads to a change of anion arrangement from a slightly distorted $h c p$ arrangement in $\mathrm{FeF}_{3}$ to a near-perfect $h c p$ arrangement in $A / B-\mathrm{Li}_{x} \mathrm{Fe}_{y} \mathrm{~F}_{3}$. Bottom: structure transition from $\mathrm{FeF}_{3}$ to the two lithiated phases, determined by HRXRD and PDF analyses. The same colours in each structure show the six equivalent $\left[\mathrm{FeF}_{6}\right]$ octahedral sites with their respective Wyckoff positions $a_{1}-a_{6}$. The white sites in $\mathrm{FeF}_{3}$ represents vacant sites. All $\mathrm{Li}$ and vertex $\mathrm{F}$ atoms are omitted for a clearer view. 

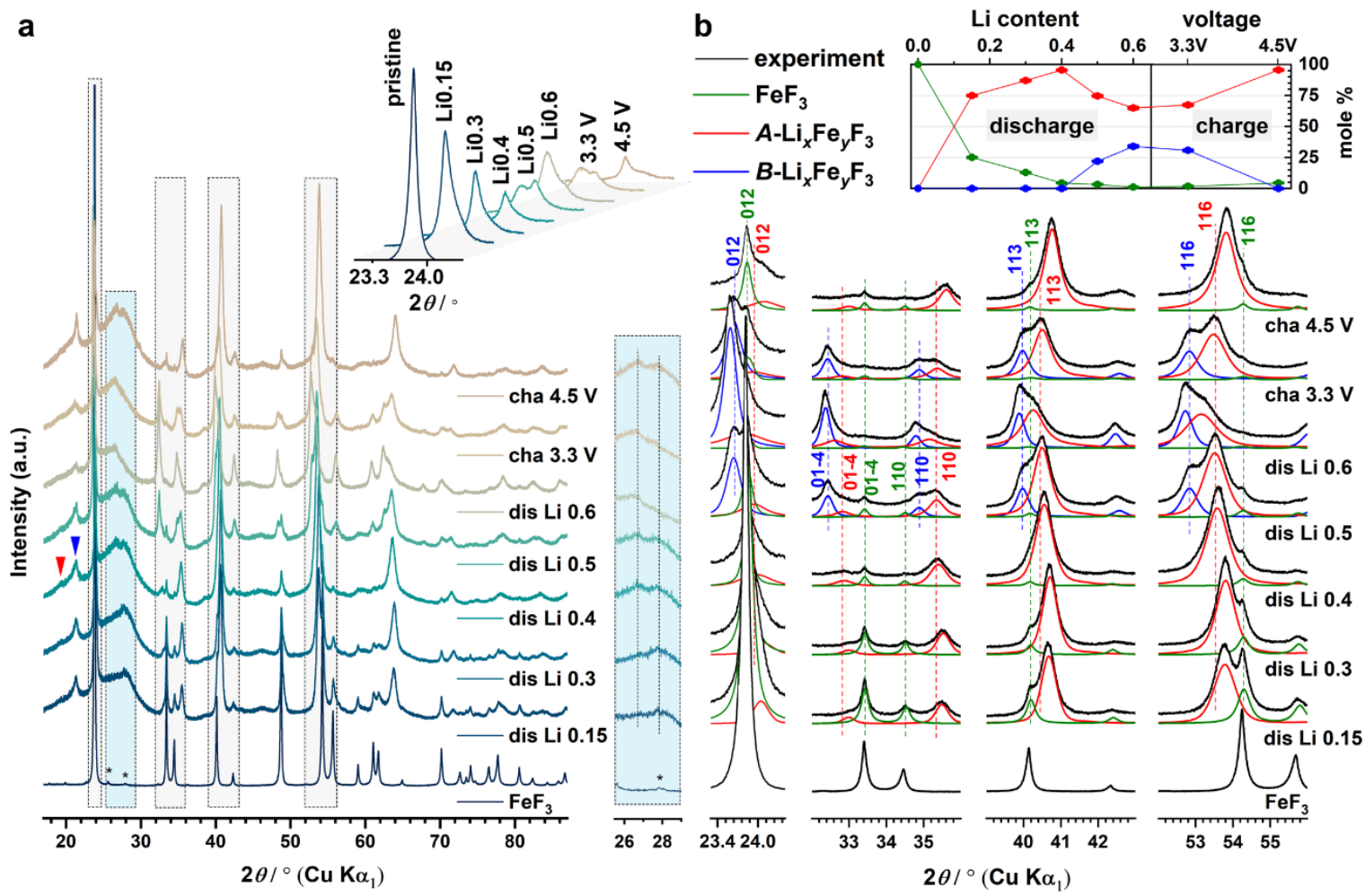

C
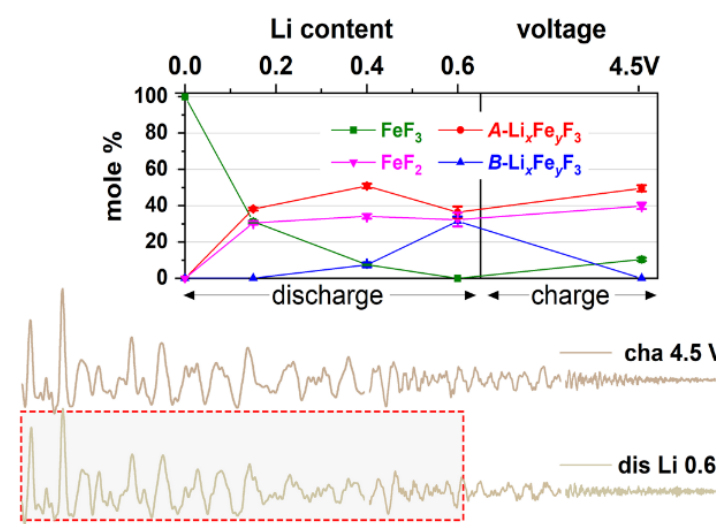

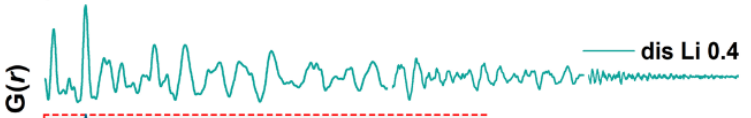

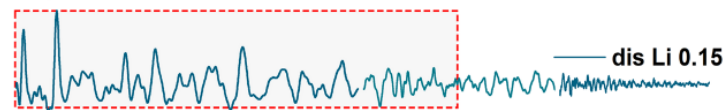
|.

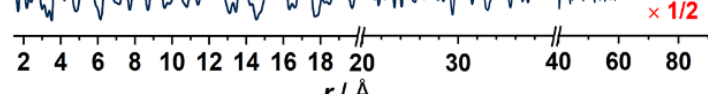

d
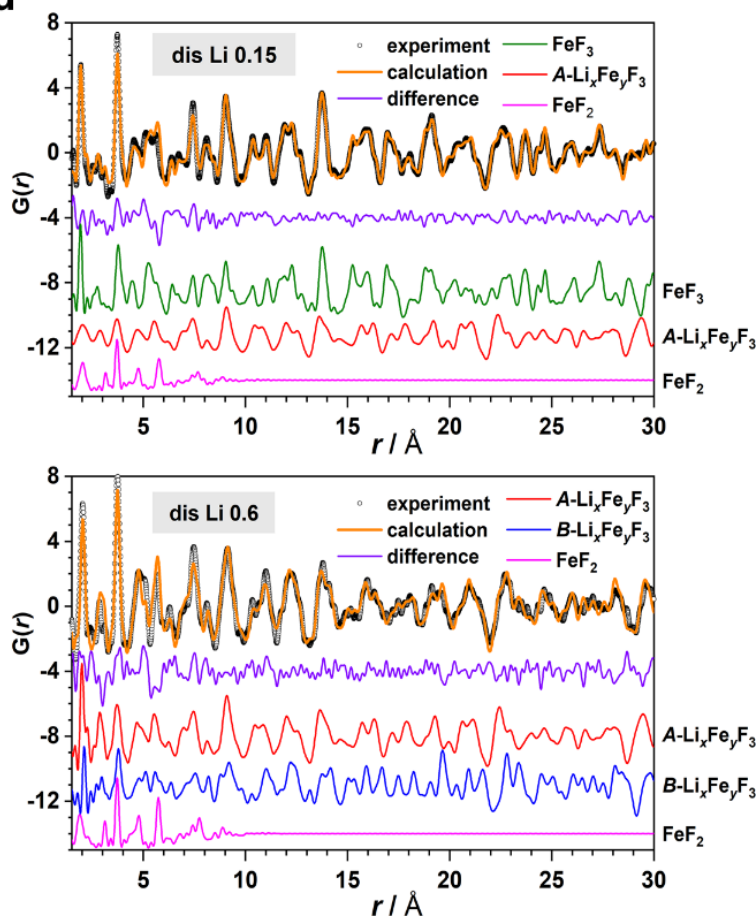

Fig. 2 XRD and PDF analysis of $\mathbf{m}-\mathbf{F e F}_{3}$. a) HRXRD data for the cycled $\mathrm{m}-\mathrm{FeF}_{3}$ collected at selected states of charge. The (003) and (01-1) reflections of the $A-\mathrm{Li}_{x} \mathrm{Fe}_{y} \mathrm{~F}_{3}$ phase are marked by red and blue triangles, respectively. Asterisks mark the hydrated $\mathrm{FeF}_{3}$ impurity introduced during sample preparation. Top inset shows the intensity variations of the (012) reflection with state of charge. Right blue inset highlights the two components in the broad feature at around $26^{\circ}-28^{\circ} 2 \theta$. b) Bragg contribution of $\mathrm{FeF}_{3}$ (green), $A-\mathrm{Li}_{x} \mathrm{Fe}_{y} \mathrm{~F}_{3}$ (red), and $B-\mathrm{Li}_{x} \mathrm{Fe}_{y} \mathrm{~F}_{3}$ (blue) derived from Rietveld refinement shown in selected $2 \theta$ range. Key reflections of each phase are marked by dashed lines with indices labelled. The inset shows the evolution of the phase mole fraction within the first insertion cycle. c) 
PDFs of $\mathrm{m}-\mathrm{FeF}_{3}$ collected within the insertion cycle. Low- $r$ atom pairs in $\mathrm{FeF}_{3}$ are labelled. Top inset shows the phase evolution of each component derived from refinement. The breakdown of the PDF contributions obtained from refinement of the PDFs from the red dashed rectangles are shown in d) where experimental, calculated and difference PDFs are shown in black circle, orange and purple lines, respectively. They are compared to the calculated PDFs from the related structures plotted using the same colour code as that in b). 

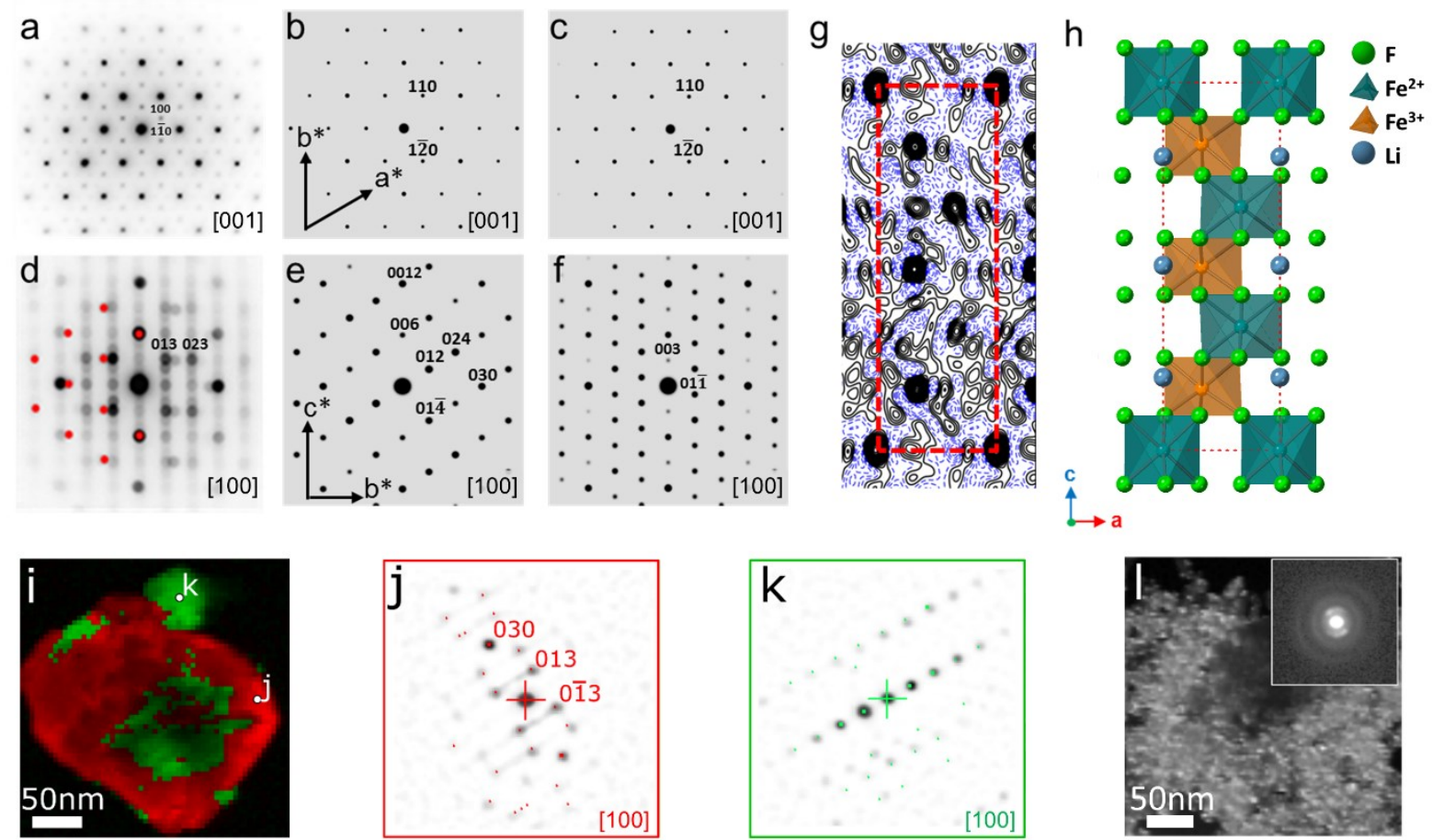

Fig. 3 | ED analyses of the partially discharged $\mathrm{m}-\mathrm{FeF}_{3}$ "Li 0.25 " sample. a - f) Zone axis ED analysis. a) The diffraction pattern recorded parallel to the [001] direction of the crystal; b) and c) are simulated ED patterns for the same orientation of $\mathrm{FeF}_{3}$ and $\mathrm{X}$-ray derived $A$ - $\mathrm{Li}_{x} \mathrm{Fe}_{y} \mathrm{~F}_{3}$, respectively. d) Experimental pattern recorded parallel to [100] (additional reflections from $\mathrm{FeF}_{2}$ in the electron beam are marked by red circles on one side of the pattern). e) and $\mathbf{f}$ ) are simulations respectively for $\mathrm{FeF}_{3}$ and $A$ - $\mathrm{Li}_{x} \mathrm{Fe}_{y} \mathrm{~F}_{3}$. g) A projected potential map solved from the pattern in d) using charge flipping. h) Structure model (P312) based on the heavy atom positions in g) and derived by DFT. i) SED analysis. The red and green colours correspond to $A-\mathrm{Li}_{x} \mathrm{Fe}_{y} \mathrm{~F}_{3}$ and $\mathrm{FeF}_{3}$, respectively. The diffraction pattern collected at position " $\mathrm{j}$ " is shown in $\mathbf{j})$ where the strong $(013) /(01-3)$ reflections are assigned to the $A$ $\mathrm{Li}_{x} \mathrm{Fe}_{y} \mathrm{~F}_{3}$ structure. The diffraction pattern collected at position " $\mathrm{k}$ ", $\mathbf{k}$ ), shows reflections consistent with the pristine $\mathrm{FeF}_{3}$ structure. l) Direct-beam intensity mapping of the sample shows significant amounts of the less-crystalline $\mathrm{FeF}_{2}$ phase, whose lack of long-range ordering is reflected by the diffraction pattern in the inset. 
a

$$
A-\mathrm{Li}_{x} \mathrm{Fe}_{y} \mathrm{~F}_{3}
$$

(faulted P312)

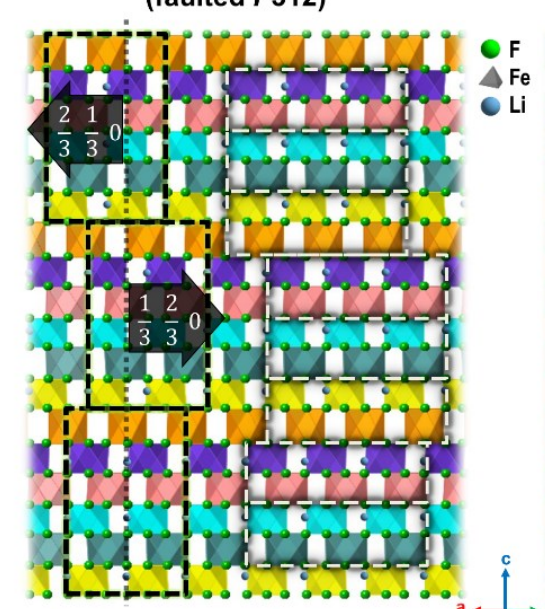

\section{b}

$\alpha-\mathrm{LiFe}^{2+} \mathrm{Fe}^{3+} \mathrm{F}_{6}$

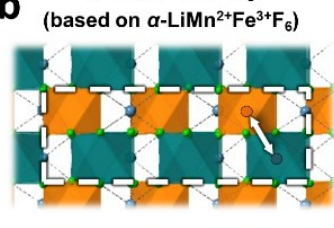

C $\quad A-\mathrm{Li}_{x} \mathrm{Fe}_{y} \mathrm{~F}_{3}$

$\left(\alpha / \beta-\mathrm{LiFe}^{2+} \mathrm{Fe}^{3+} \mathrm{F}_{6}\right)$

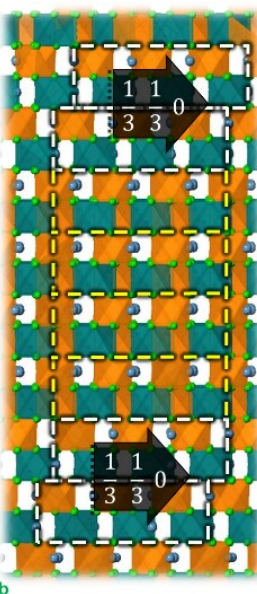

$\beta$ - $\mathrm{LiFe}^{2+} \mathrm{Fe}^{3+} \mathrm{F}_{6}$

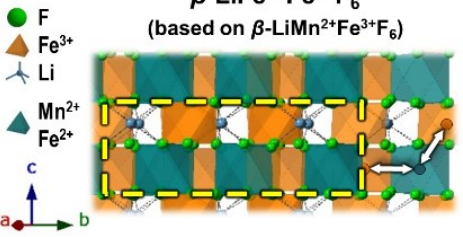

d

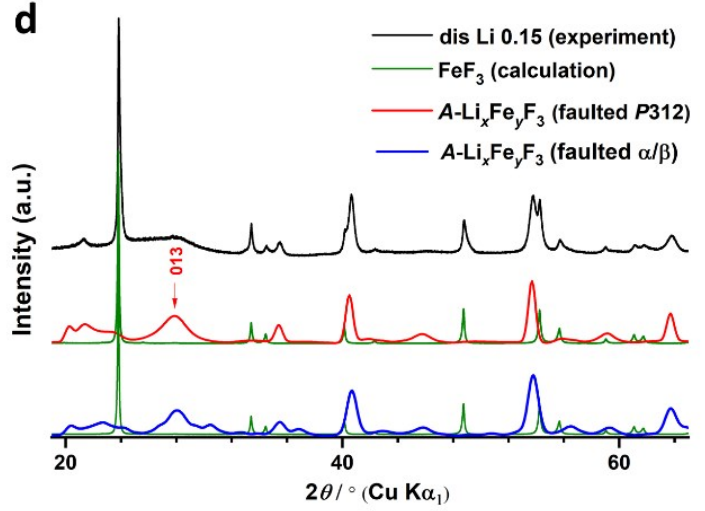

e
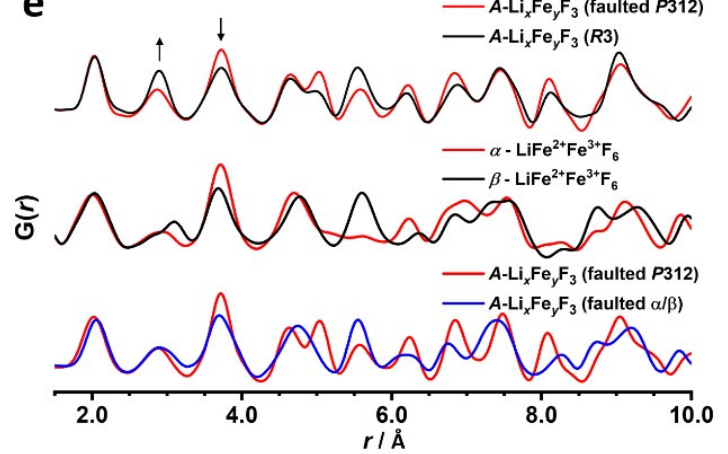

Fig. 4 Stacking disorder in $\boldsymbol{A}-\mathrm{Li}_{x} \mathbf{F e}_{y} \mathbf{F}_{3}$. a) Faulted $A-\mathrm{Li}_{x} \mathrm{Fe}_{y} \mathrm{~F}_{3}$ model constructed by stacking the ED-derived $P 312$ cells (black dashed rectangles) along the $c$-axis with displacement vectors shown. White dashed rectangles indicate an alternative structure representation based on the $\mathrm{LiMnFeF}_{6}$ structure. b) Structures of $\alpha-\mathrm{LiFe}_{2} \mathrm{~F}_{6}$ (white arrows indicate corner shared $\left[\mathrm{MF}_{6}\right] ; \mathrm{M}-\mathrm{Fe}^{3+}, \mathrm{Fe}^{2+}$ and $\mathrm{Mn}^{2+}$ in the related $\mathrm{LiM}^{2+} \mathrm{M}^{3+} \mathrm{F}_{6}$ structure) and $\beta$ - $\mathrm{LiFe}_{2} \mathrm{~F}_{6}$ (white arrows indicate corner shared and edge shared $\left.\left[\mathrm{MF}_{6}\right]\right)$. c) Structure of $A-\mathrm{Li}_{x} \mathrm{Fe}_{y} \mathrm{~F}_{3}$ composed of disordered stacking of $\alpha$ - and $\beta$ - $\mathrm{LiFe}_{2} \mathrm{~F}_{6}$ units (marked by white and yellow dashed rectangle respectively) with displacement vectors indicated. d) XRD comparison between the experiment, calculated $\mathrm{FeF}_{3}$ and the $A-\mathrm{Li}_{x} \mathrm{Fe}_{y} \mathrm{~F}_{3}$ patterns. The $A-\mathrm{Li}_{x} \mathrm{Fe}_{y} \mathrm{~F}_{3}$ patterns were simulated using the faulted model in a) and c), showing a comparable result. e) Top: PDF comparison between faulted $P 312$ model and the average structure $(R 3)$ with black arrows indicating the local structure difference, which is comparable to that between $\alpha$ - and $\beta$ - $\mathrm{LiFe}_{2} \mathrm{~F}_{6}$ shown in the middle. Bottom: PDF comparison between simulations using the faulted models shown in a) and c). 

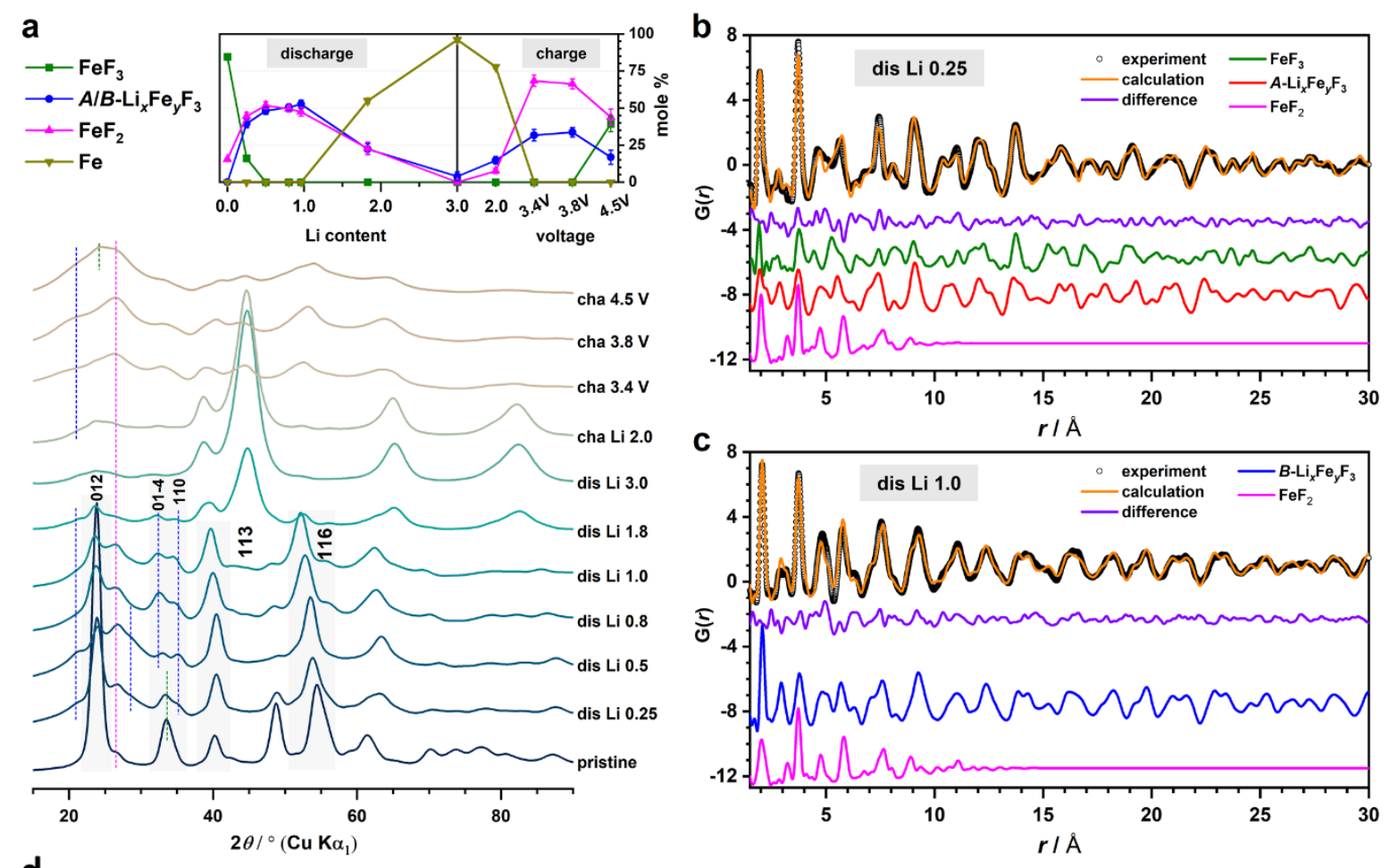

d

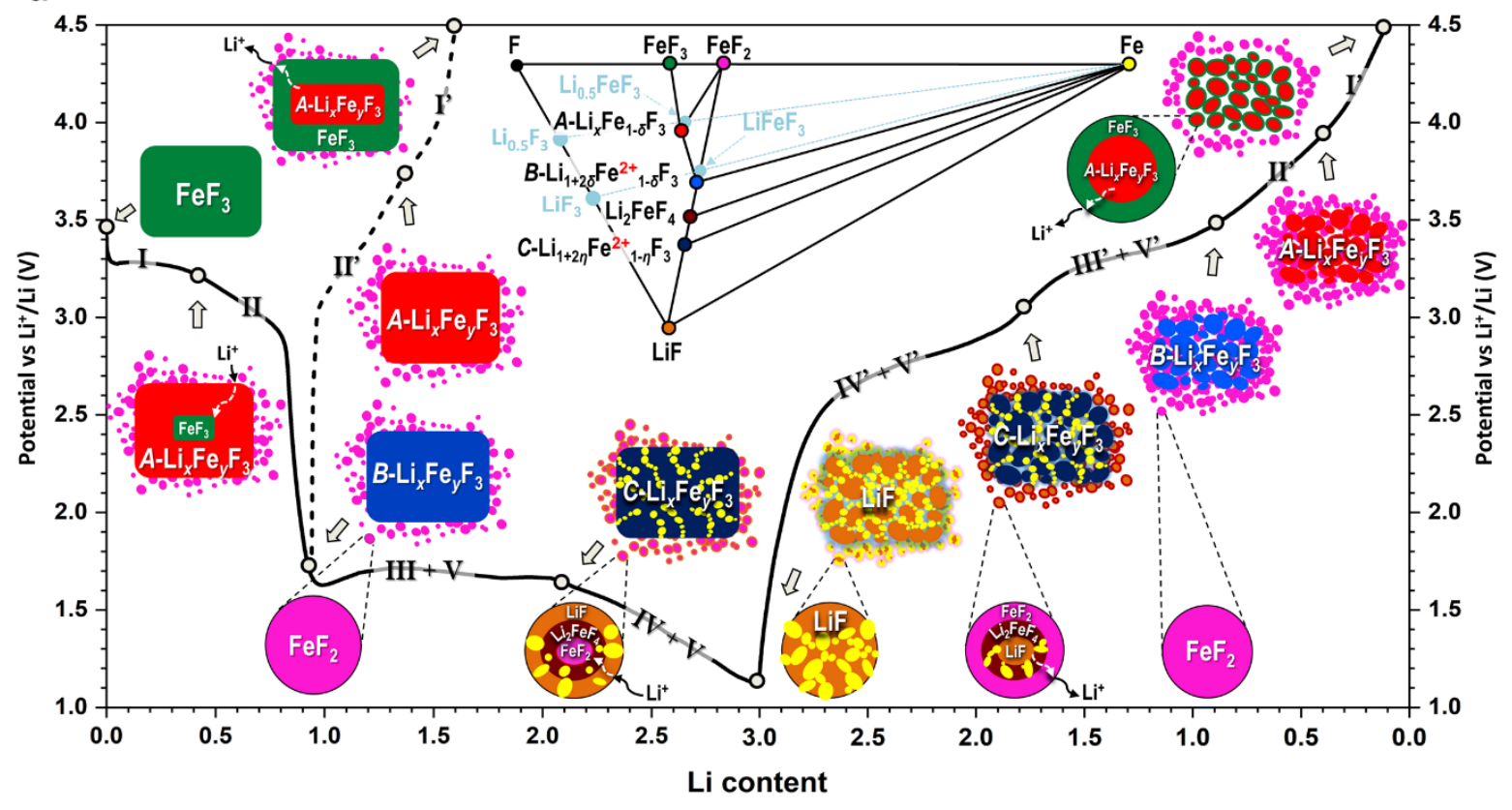

Fig. 5 | XRD and PDF of $n-\mathrm{FeF}_{3}$ and illustrated reaction pathways for $\mathrm{FeF}_{\mathbf{x}}$. a) XRD data obtained from X-ray total scattering experiment of selected $n-\mathrm{FeF}_{3}$ samples. Structural features that highlight the similarity with the data obtained for $\mathrm{m}_{-} \mathrm{FeF}_{3}$ in Fig. 2a are shaded and indexed. Bragg peaks unique to $\mathrm{FeF}_{3}$ (green), $A / B-\mathrm{Li}_{x} \mathrm{~F}_{y} \mathrm{~F}_{3}$ (blue), $\mathrm{FeF}_{2}$ (pink) and $\mathrm{Fe}$ (dark yellow) are marked by dotted lines. Inset shows phase evolution of these phases derived from refinement of the corresponding PDF data. The presence of $\mathrm{FeF}_{2}$ in the pristine sample results from the reduction of $\mathrm{FeF}_{3}$ via ball-milling, during which carbon is fluorinated to form $\mathrm{CF}_{x}^{41}$. The refinement results of b) "dis Li 0.25 " and c) "dis Li 1.0" n$\mathrm{FeF}_{3}$ are broken down into the patterns from the individual phases to highlight the similar phase behaviour seen during lithiation for $n-$ and $m-\mathrm{FeF}_{3}$ (latter shown in Fig. 2d). d) Simplified Li-Fe-F ternary phase diagram (see Extended Data Fig. 2 for the full diagram) and illustration of reaction pathways of the $\mathrm{FeF}_{3}-\mathrm{FeF}_{2}$ system (with voltage curves for insertion charge and full reaction cycle 
respectively denoted using dashed and solid lines). The reference phases in the phase diagram are labelled and indicated by light blue circles to indicate the positions of the $A$ - and $B$ - $\mathrm{Li}_{x} \mathrm{Fe}_{y} \mathrm{~F}_{3}$, whose $\mathrm{Fe}$ concentration is off-stoichiometric. The colour of each phase in the phase diagram is consistent with those in the pathway illustration. Impeded internal and external Li transport is indicated by black solid and white dashed arrows, respectively. 
a

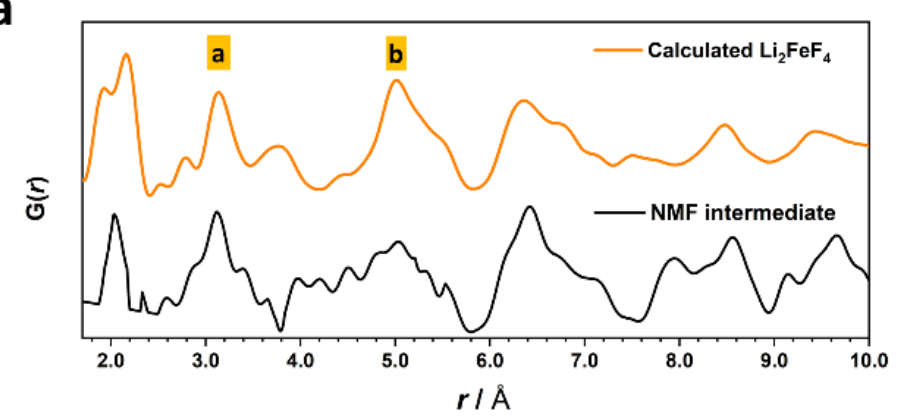

b

$\mathrm{FeF}_{2}$

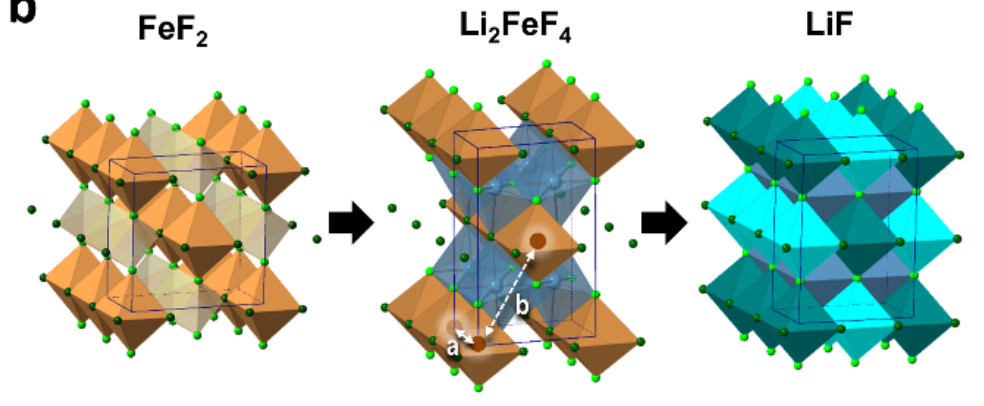

$\mathrm{Li}_{2} \mathrm{FeF}_{4}$

LiF

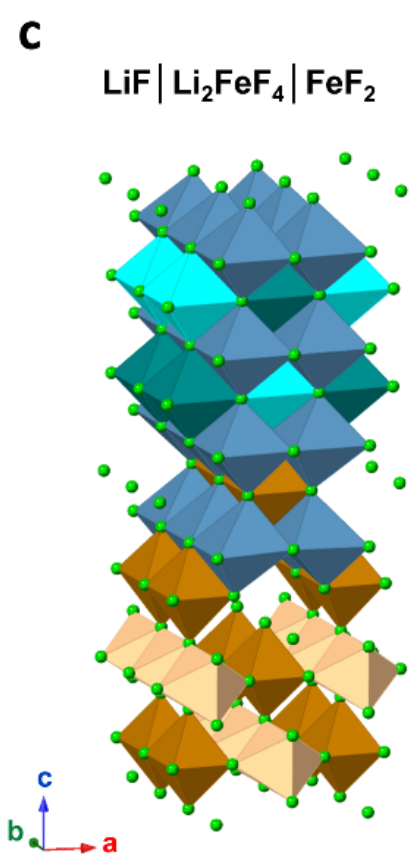

Fig. 6 | Formation of interfacial intermediate upon lithiation of $\mathbf{F e F}_{2}$. a) PDF comparison between the calculated pattern and the NMF-derived pattern for $\mathrm{Li}_{2} \mathrm{FeF}_{4}(\mathrm{Cmmm})$. b) The structures of the reactant $\left(\mathrm{FeF}_{2}\right)$, intermediate $\left(\mathrm{Li}_{2} \mathrm{FeF}_{4}\right)$ and product $(\mathrm{LiF})$. To highlight a topological relationship between these phases, these structures are represented by using the unit cell and symmetry setting of $\mathrm{Li}_{2} \mathrm{FeF}_{4}$. In each phase, the atoms that are equivalent based on the Cmmm symmetry are depicted using the same colour. The two unique $\mathrm{Fe}-\mathrm{Fe}$ atom pairs in $\mathrm{Li}_{2} \mathrm{FeF}_{4}$ are indicated by "a" and " $b$ " in the structure and PDF pattern. c) " $\mathrm{LiF}\left|\mathrm{Li}_{2} \mathrm{FeF}_{4}\right| \mathrm{FeF}_{2}$ " interface model, which contains an interfacial $\mathrm{Li}_{2} \mathrm{FeF}_{4}$ block coherently connected to $\mathrm{LiF}$ and $\mathrm{FeF}_{2}$ units based on their symmetry relationships, shown using the same colour code used in b). 

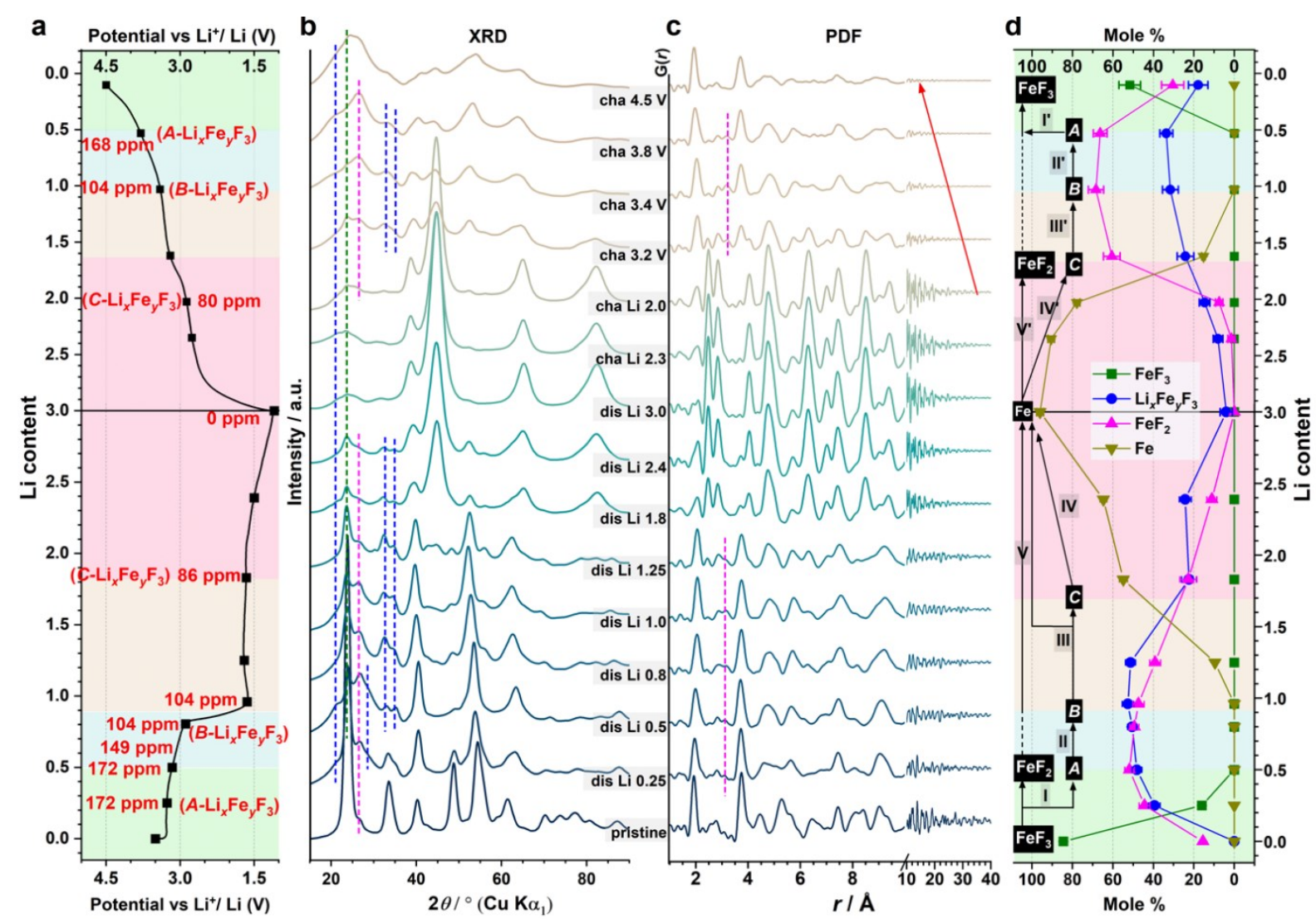

Extended Data Fig. 1 NMR, XRD and PDF results of $\mathbf{n}-\mathrm{FeF}_{3}$ in the first cycle. a) Galvanostatic profile of $n-\mathrm{FeF}_{3}$ 's first cycle with distinct reaction processes indicated by different colour backgrounds. The ${ }^{6} \mathrm{Li}$ MAS NMR chemical shifts (red) from our previous study ${ }^{10}$ are labelled at their respective state of charge with the main phase identification indicated. Black squares mark the states of charge where ex situ X-ray total scattering experiments were performed to acquire b) XRD and c) PDF patterns. Green, blue and pink dotted lines indicate unique Bragg or PDF features from $\mathrm{FeF}_{3}, \mathrm{Li}_{x} \mathrm{Fe}_{y} \mathrm{~F}_{3}$ and $\mathrm{FeF}_{2}$, respectively. The red arrow highlights a drastic decrease in the samples' particle sizes upon charge. The evolution of the phase mole fractions obtained from the PDF refinement is shown in d) with the deduced step-by-step mechanism diagram indicated on the left, which echoes with the simplified scheme shown in Fig. 5d. 


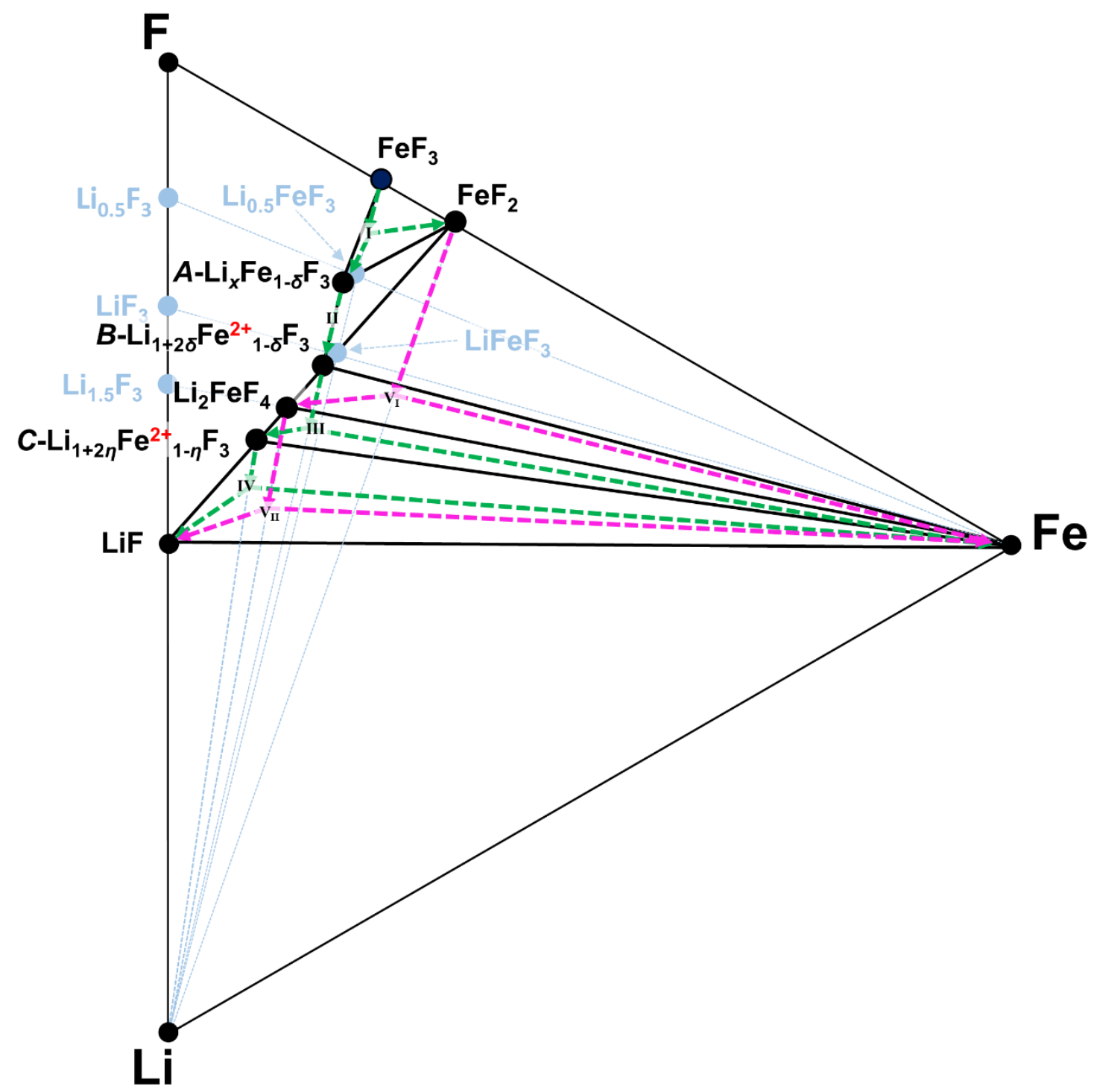

Extended Data Fig. 2 Li-Fe-F phase diagram. The reference phases in the phase diagram are labelled and indicated by light blue circles so as to indicate the positions of $A$ - and $B-\mathrm{Li}_{x} \mathrm{Fe}_{y} \mathrm{~F}_{3}$, whose $\mathrm{Fe}$ concentration is off-stoichiometric. The reaction pathways associated with the $\mathrm{FeF}_{3}$ and $\mathrm{FeF}_{2}$ systems are respectively marked by using green and pink dashed arrows. Each reaction process is also labelled with its respective roman numeral used to label the equations in the manuscript. 